\title{
STABILITY OF EQUILIBRIA FOR THE STEFAN PROBLEM WITH SURFACE TENSION
}

\author{
JAN PRÜSS AND GIERI SIMONETT
}

\begin{abstract}
We characterize the equilibrium states for the two-phase Stefan problem with surface tension and with or without kinetic undercooling, and we analyze their stability in dependence of physical and geometric quantities.
\end{abstract}

\section{INTRODUCTION}

The Stefan problem is a model for phase transitions in solid-liquid systems. In this paper, we consider the two-phase Stefan problem with the modified GibbsThomson law

$$
u=\sigma H+\delta V \quad \text { on } \quad \Gamma(t), \quad \sigma>0, \quad \delta \geq 0,
$$

and the kinetic condition

$$
\left[d \partial_{\nu} u\right]=(\ell-[\kappa] u) V \quad \text { on } \quad \Gamma(t) .
$$

Here $\Gamma(t)$ denotes the unknown moving hypersurface that separates the liquid from the solid phase, $u$ is the temperature, $H$ the mean curvature of $\Gamma(t), \sigma$ the surface tension coefficient, $\delta$ the coefficient of kinetic undercooling, $V$ the normal velocity of $\Gamma(t), \ell$ the latent heat, $[\kappa]$ the jump of the heat capacities across $\Gamma(t)$, and $\left[d \partial_{\nu} u\right]$ the jump of the heat fluxes across $\Gamma(t)$. Note that in case $\sigma=\delta=0$, i.e. for the classical Stefan problem, we have $u=0$ at the interface, and then the kinetic condition becomes the classical Stefan condition.

Under appropriate boundary conditions we will show that spheres (together with constant temperature distributions) are the only equilbrium states for this system, and we will characterize the stability of these equilibria in dependence of physical and geometric quantities.

In order to formulate the Stefan problem we introduce the following notations. Let $\Omega$ be a smooth bounded domain in $\mathbb{R}^{n}$ whose boundary $\partial \Omega$ consists of two disjoint components, an 'interior' part $J_{1}$ and an 'exterior' part $J_{2}$. We think of $\Omega$ as a homogeneous medium which is occupied by a liquid and a solid phase, say water and ice, that initially occupy the regions $\Omega_{0}^{1}$ and $\Omega_{0}^{2}$, and that are separated by a sharp interface $\Gamma_{0}$. More precisely, we assume that $\Gamma_{0} \subset \Omega$ is a compact closed hypersurface, that $\Omega_{0}^{1}$ and $\Omega_{0}^{2}$ are disjoint open sets such that $\bar{\Omega}=\overline{\Omega_{0}^{1}} \cup \overline{\Omega_{0}^{2}}$ and such that $\partial \Omega_{0}^{i}=J_{i} \cup \Gamma_{0}$ for $i=1,2$. For the sake of definiteness we consider the open set $\Omega_{0}^{1}$ as the region occupied by the liquid phase. Consequently, the

The research of the second author was partially supported by NSF, Grant DMS-0600870. 
component $J_{1}$ is in contact with the liquid phase and $J_{2}$ is in contact with the solid phase. The boundaries $J_{1}$ and $J_{2}$, corresponding for instance to the walls of a container, are fixed, whereas $\Gamma_{0}$ will change as time evolves, due to solidification or liquidation of the two different phases.

Given $t \geq 0$, let $\Gamma(t)$ be the position of $\Gamma_{0}$ at time $t$, and let $V(\cdot, t)$ and $H(\cdot, t)$ be the normal velocity and the mean curvature of $\Gamma(t)$. Moreover, let $\Omega_{1}(t)$ and $\Omega_{2}(t)$ be the two regions in $\Omega$ separated by $\Gamma(t)$. According to our assumption, $\Omega_{1}(t)$ is the region occupied by the liquid phase, and $\Gamma(t)$ is a sharp interface which separates the liquid from the solid phase. Let $\nu(\cdot, t)$ be the outer unit normal field on $\Gamma(t)$ with respect to $\Omega_{1}(t)$. We shall use the convention that the normal velocity is positive if $\Omega_{1}(t)$ is expanding, and that the mean curvature is positive if the intersection of $\Omega_{1}(t)$ with a small ball centered at $\Gamma(t)$ is convex. Consequently, the normal velocity is positive if the liquid region is growing, $\nu$ points into the solid phase, and $H$ is positive for a water ball surrounded by ice, and negative for an ice ball surrounded by water.

Here we concentrate on the case $J_{1}=\emptyset$. Let $\Gamma_{0}$ and $u_{0}^{i}: \Omega_{0}^{i} \rightarrow \mathbb{R}$ be given, where $u_{0}^{1}$ and $u_{0}^{2}$ denote the initial temperatures of the liquid and solid phase, respectively. The strong formulation of the two-phase Stefan problem with surface tension and kinetic undercooling consists of finding a family $\Gamma:=\{\Gamma(t) ; t \geq 0\}$ of hypersurfaces and functions $u_{i}: \cup_{t \geq 0}\left(\Omega_{i}(t) \times\{t\}\right) \rightarrow \mathbb{R}$, satisfying

$$
\left\{\begin{aligned}
\kappa_{i} \partial_{t} u_{i}-d_{i} \Delta u_{i} & =0 & & \text { in } \Omega_{i}(t) \\
\partial_{\nu} u_{2} & =0 & & \text { on } J_{2} \\
u_{i} & =\sigma H_{\Gamma}+\delta V & & \text { on } \Gamma(t) \\
{\left[d \partial_{\nu} u\right] } & =(\ell-[\kappa] u) V & & \text { on } \Gamma(t) \\
u^{i}(0) & =u_{0}^{i} & & \text { in } \Omega_{0}^{i} \\
\Gamma(0) & =\Gamma_{0}, & &
\end{aligned}\right.
$$

where $\kappa_{i} \geq 0$ is the heat capacity of phase $i, d_{i}$ its thermal conductivity coefficient, $\ell>0$ is the latent heat per unit mass absorbed or released for melting or solidifying, $\sigma>0$ is the surface tension, and $\delta \geq 0$ is the speed of kinetic undercooling. Moreover,

$$
\begin{aligned}
{[\kappa]: } & =\kappa_{2}-\kappa_{1}, \\
{\left[d \partial_{\nu} u\right]: } & =d_{2} \partial_{\nu} u_{2}-d_{1} \partial_{\nu} u_{1}
\end{aligned}
$$

denote the jump of the heat capacities and the heat fluxes, respectively, across the interface $\Gamma(t)$. Note $[\kappa]=\kappa_{2}-\kappa_{1}<0$ is physically reasonable since in the liquid phase there are more degrees of freedom than in the solid phase, hence the liquid phase can absorb more energy per unit mass. However, we do not assume $[\kappa]<0$ in the sequel. The condition $u_{i}=\sigma H_{\Gamma}$ on the free interface is usually called the Gibbs-Thomson law, and $u_{i}=\sigma H_{\Gamma}+\delta V$ the modified Gibbs-Thomson law, or the Gibbs-Thomson law with kinetic undercooling; see [2, 3, 18, 19, 21, 23, 26, 27, 32] for more information. 
We refer to $[12,13,16,24,25,28,29]$ for existence and regularity results for the Stefan problem with the Gibbs-Thomson law $u_{i}=\sigma H_{\Gamma}$ in case $\kappa_{1}=\kappa_{2}$. The Stefan problem with surface tension and kinetic undercooling in case $\kappa_{1}=\kappa_{2}$ has been studied in [5, 28, 29, 31], see also [22] for the one-phase case.

It will be shown in [14] that the Stefan problem (1.3) has a unique local solution which is analytic in space and time, provided the well-posedness condition

$$
\ell-\sigma[\kappa] H_{\Gamma_{0}}>0 \text { in case } \delta=0
$$

is satisfied. On the other hand, if $\delta=0$ and $\kappa_{1}>\kappa_{2}$, problem (1.3) is not wellposed if $H_{\Gamma_{0}}$ is too negative, that is, in case the solid region sharply protrudes into the liquid. Associated to the Stefan problem (1.3) is the energy functional

$$
E(u(t), \Gamma(t)):=\int_{\Omega} \kappa u d x+\ell\left|\Omega_{1}(t)\right|=\int_{\Omega_{1}(t)} \kappa_{1} u_{1} d x+\int_{\Omega_{2}(t)} \kappa_{1} u_{2} d x+\ell\left|\Omega_{1}(t)\right|,
$$

where $\left|\Omega_{1}(t)\right|$ is the volume of the region $\Omega_{1}(t)$. If $(u, \Gamma)$ is a sufficiently smooth solution of (1.3) then we obtain

$$
\begin{aligned}
\frac{d}{d t} E(u(t), \Gamma(t)) & =\int_{\Omega_{1}(t)} \kappa_{1} \partial_{t} u_{1} d x+\int_{\Omega_{2}(t)} \kappa_{1} \partial_{t} u_{2} d x-[\kappa] \int_{\Gamma(t)} u V d s+\ell \int_{\Gamma(t)} V d s \\
& =\int_{\Gamma(t)}\left(-\left[d \partial_{\nu} u\right]-[\kappa] u V+\ell V\right) d s=0,
\end{aligned}
$$

thus showing that energy is conserved.

If $\kappa_{1}=\kappa_{2}=0$ and $\delta=0$, then the resulting problem is the quasi-stationary Stefan problem with surface tension, which has also been termed the Mullins-Sekerka model (or the Hele-Shaw model with surface tension). Existence, uniqueness, regularity, and global existence of solutions for the quasi-stationary approximation has been investigated in $[1,4,6,8,9,10,11,17]$. Existence and global existence of classical solutions for the quasi-stationary approximation with $\sigma>0$ and $\delta>0$ has been studied in [33, 22].

A major difficulty in the mathematical treatment of the Stefan problem (1.3) is due to fact that the boundary $\Gamma(t)$, and thus the geometry, is unknown and ever changing. A widely used method to overcome this inherent difficulty is to choose a fixed reference surface $\Sigma$ and then represent the moving surface $\Gamma(t)$ as the graph of a function (which we will denote by $\rho=\rho(s, t)$ ) in normal direction of $\Sigma$. In this way, one obtains a time-dependent (unknown) diffeomorphism from $\Sigma$ onto $\Gamma(t)$, and in a next step this diffeomorphism is extended to a diffeomorphism of fixed reference regions $D^{i}$ onto the unknown domains $\Omega_{i}(t)$. The treatment of the moving boundary problem (1.3) then proceeds by transforming the equations into a new system of equations defined on the fixed domain $D_{1} \cup D_{2}$ from which both the solution and the parameterizing function $\rho$ have to be determined. In the context of the Stefan problem this approach has first been used by Hanzawa [20]. 
The same approach has also been used in $[10,11]$ for the quasi-stationary approximation of the Stefan problem with surface tension, and in [14] for the Stefan problem with surface tension. Once the transformed system has been obtained, one can study the mapping properties of the nonlinearities involved, and in particular, one can determine their linearizations, see [14] for more details.

In this paper, we assume that $\Gamma(t)$ does not touch the fixed boundary $J_{2}=\partial \Omega$. Under this assumption, we will characterize all the equilibrium states $\left(u_{1}, u_{2}, \Sigma\right)$ of (1.3). In fact, it is easy to see that the equilibria are precisely given by

$$
\Sigma=\bigcup_{j=1}^{m} S_{R}\left(x_{j}\right), \quad u_{1}=u_{2}=\sigma / R
$$

where $S_{R}\left(x_{j}\right)$ denote disjoint spheres of the same radius $R$ and centers $x_{j}$. This can be seen by the following arguments: the equilibria $\left(u_{1}, u_{2}, \Sigma\right)$ of the Stefan problem (1.3) are given by the system of equations

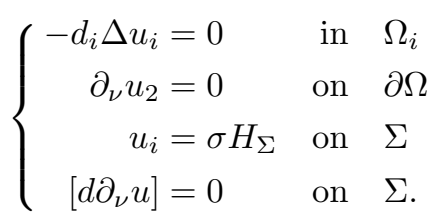

Taking the inner product of $(1.7)_{1}$ with $u_{i}$, the divergence theorem and condition $(1.7)_{4}$ yield

$$
\int_{\Omega_{i}}\left|\nabla u_{i}\right|^{2} d x=0
$$

hence $u_{i}$ is constant on $\Omega_{i}$. Equation $(1.7)_{3}$, in turn, shows that $u_{1}=u_{2}$ and also that $H=u / \sigma$ is constant on $\Sigma$. But then, since $\Omega$ is bounded, $\Sigma$ must be a sphere $S_{R}\left(x_{0}\right)$ centered at some point $x_{0} \in \Omega$ with radius $R>0$, if the phases are connected. Otherwise, again due to the boundedness of $\Omega, \Sigma$ is the union of finitely many spheres of the same radius $R>0$. Here we concentrate on the case of connected phases. Thus there is an $(n+1)$-parameter family of equilibria, the parameters being the $n$ coordinates of the center $x_{0}$ and the radius $R$.

We want to discuss the stability of those equilibria. The linearized problem (associated to the transformed equations) at such an equilibrium state is given by

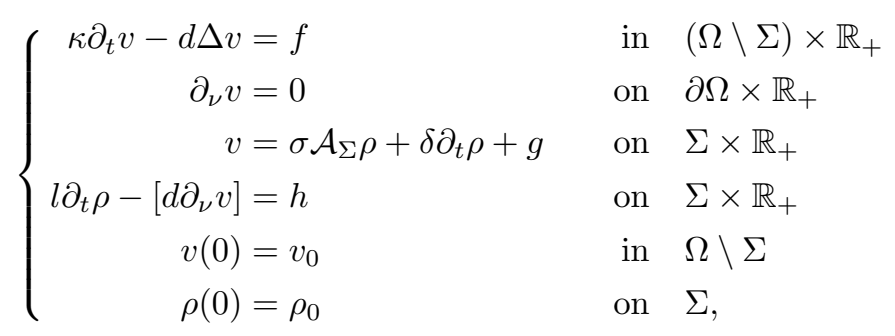


see [14]. Here, $l=\ell-[\kappa] \sigma / R$, and the operator $\mathcal{A}_{\Sigma}$ is given by

$$
\mathcal{A}_{\Sigma}=-\frac{1}{n-1}\left(\frac{n-1}{R^{2}}+\Delta_{\Sigma}\right)
$$

where $\Delta_{\Sigma}$ denotes the Laplace-Beltrami operator on $\Sigma$. This is the linearization of the mean curvature $H^{\prime}(0)$ at the sphere $\Sigma$; cf. e.g. Escher and Simonett [11]. Here we use the notation $v=v_{1} \chi_{\Omega_{1}}+v_{2} \chi_{\Omega_{2}}$, where $\chi_{G}$ denotes the characteristic function of a set $G$, and similarly $\kappa=\kappa_{1} \chi_{\Omega_{1}}+\kappa_{2} \chi_{\Omega_{2}}$ and $d=d_{1} \chi_{\Omega_{1}}+d_{2} \chi_{\Omega_{2}}$. Associated to the linearization (1.8) is the following eigenvalue problem

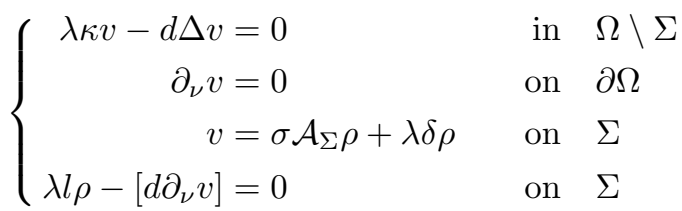

where as before $l=\ell-[\kappa] \sigma / R$. We will now state the main results of this paper. We will formulate our results for a domain in $\mathbb{R}^{n}$ for $n \in \mathbb{N}, n>1$, although the physically relevant dimensions, naturally, are $n=2,3$.

Theorem 1.1. Suppose that the phases in the Stefan problem are connected. Then

(a) The equilibrium states (without boundary contact) for problem (1.3) are given by

$$
(u, \Sigma), \quad \text { where } \Sigma=S_{R}\left(x_{0}\right) \text { and } u=\sigma / R,
$$

with $S_{R}\left(x_{0}\right) \subset \Omega$ being the sphere with radius $R$ and center $x_{0}$.

(b) For $l>0$, the eigenvalue problem (1.9) has countably many real eigenvalues of finite algebraic multiplicity.

(c) 0 is an eigenvalue of (1.9) with geometric multiplicity $(n+1)$. The (geometric) eigenspace is spanned by

$$
\left(-1, Y_{0}\right),\left(0, Y_{1}\right), \ldots,\left(0, Y_{n}\right),
$$

where $Y_{0}=R^{2} / \sigma$, and where $Y_{j}, 1 \leq j \leq n$, are the spherical harmonics of degree 1 (normalized by the orthogonality condition $\left(Y_{i} \mid Y_{j}\right)_{\Sigma}=\delta_{i j}$ ).

(d) If $\sigma(\kappa \mid 1)_{\Omega} \leq l|\Sigma| R^{2}$, then (1.9) has no positive eigenvalues.

(e) If $\sigma(\kappa \mid 1)_{\Omega}>l|\Sigma| R^{2}>0$, then (1.9) has exactly one positive, algebraically simple eigenvalue.

(f) If $l<0$ and $\delta>0$, then (1.9) has at least one positive eigenvalue.

(g) If $l<0$ and $\delta=0$, then the linearized problem (1.8) is not well-posed.

Proof. The assertion in (a) has been proved above. We refer to Theorem 2.1 for a proof of assertions (b)-(e), and for additional information about the eigenvalue problem (1.9), for the case $l>0$. The proof of (f) is given at the end of Section 5 , and $(\mathrm{g})$ follows from $[7]$. 
Remarks 1.2. (a) If $l<0$ then all equilibrium states are linearly unstable (and the linearized problem (1.8) is not even well-posed in case $\delta=0$ ). Therfore we mainly concentrate on the case $l>0$. Define then

$$
\zeta:=\frac{\sigma(\kappa \mid 1)_{\Omega}}{l|\Sigma| R^{2}}, \quad \text { where } \quad l=\ell-\frac{\sigma[\kappa]}{R}, \quad(\kappa \mid 1)_{\Omega}:=\int_{\Omega} \kappa d x .
$$

According to Theorem 1.1.(d)-(e), we know that all eigenvalues of (1.9) are nonpositive if $\zeta \leq 1$, and that there exists exactly one positive simple eigenvalue if $\zeta>1$. We will refer to the case $\zeta \leq 1$ as a stability condition.

Observe that neither the thermal conductivity coefficients $d_{i}$ nor the kinetic coefficient $\delta$ does enter this stability condition, it depends only on the heat capacities $\kappa_{i}$, the latent heat $\ell$, the surface tension $\sigma$, and on the geometry. In particular, decreasing the size of a ball decreases its stability, as does increasing surface tension, see also Remark 1.5.(a). We also mention that the stability condition $\zeta \leq 1$ is always valid in the quasi-stationary case $\kappa_{i}=0$, i.e. for the Mullins-Sekerka problem.

(b) It will be shown in the forthcoming paper [15] that solutions for the Stefan problem (1.3) that start out close to an equilibrium $(u, \Sigma)$ exist globally, and converge towards an equilibrium state $\left(u^{\prime}, \Sigma^{\prime}\right)$ as time goes to infinity, provided that $l>0$ and $\zeta<1$. This gives justice to the wording stability condition for the case $\zeta<1$. We note again that $\zeta=0$ if the heat capacities $\kappa_{i}$ are zero, that is, in the quasi-stationary case. In this case, global existence and convergence to equilibria was obtained in $[11,22]$ by using a center-manifold analysis, see also [17] for a different approach in the one-phase case.

(c) If the Gibbs-Thomson condition on the free interface $\Gamma(t)$ is replaced by $u_{i}=0$, then (1.3) is called the (classical) Stefan model. It should be observed that, in contrast to the problem with surface tension, the classical Stefan problem does not admit nontrivial equilibrium states.

For $l>0$, the results in Theorem 1.1 suggest that one eigenvalue, $\lambda_{*}$, crosses the imaginary axis at 0 as the quantity $\zeta$ increases and exceeds 1 . According to part (c) of Theorem 1.1, 0 is always an eigenvalue with geometric multiplicity $(n+1)$. This suggests that as the eigenvalue $\lambda_{*}$ crosses through 0 , the algebraic multiplicity of 0 raises by one, and then drops again as soon as the eigenvalue has crossed. This is exactly what happens, as will be proved in Theorem 2.1.

Another way to view and understand this situation can be gained from considering the following parameter-dependent eigenvalue problem

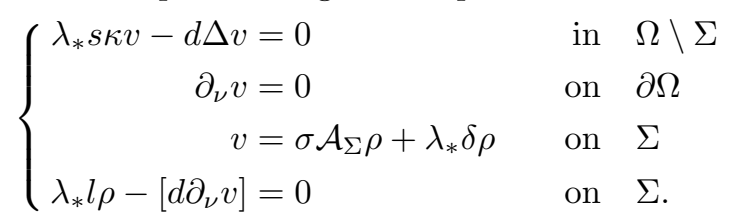

The following result will be proved in Section 6 . 
Theorem 1.3. Let $l>0$ and set $s_{0}:=l|\Sigma| R^{2} / \sigma(\kappa \mid 1)_{\Omega}$. Then

(a) The eigenvalue problem (1.10) has an analytic curve of solutions

$$
\left[s \mapsto\left(\lambda_{*}(s), v(s), \rho(s)\right)\right], \quad s \in\left(s_{0}-\varepsilon_{0}, \infty\right)
$$

such that $\lambda_{*}(s)>0$ iff $s>s_{0}$, where $\varepsilon_{0}$ is an appropriate positive number.

(b) $\lambda_{*}(s)$ crosses the imaginary axis with positive speed at $s=s_{0}$.

(c) $\left[s \mapsto \lambda_{*}(s)\right]$ is strictly increasing.

(d) If $\delta>0$ then $\lambda_{*}(s)$ is bounded above by $\sigma / \delta R^{2}$.

(e) If $\delta=0$ then $\lambda_{*}(s) \rightarrow \infty$ as $s \rightarrow \infty$.

Clearly, the eigenvalues of the modified problem (1.10) coincide with the eigenvalues of (1.9) if $s=1$. In case $\zeta>1$ we have $s_{0}<1$ and we see that $\lambda=\lambda_{*}(1)$ is a (the only) positive eigenvalue of (1.9).

According to (1.5) an equilibrium state $\left(\sigma / R, S_{R}\left(x_{0}\right)\right)$ for the Stefan problem (1.3) has energy

$$
\begin{aligned}
\phi(R): & =E\left(\frac{\sigma}{R}, S_{R}\left(x_{0}\right)\right)=\frac{\sigma}{R}(\kappa \mid 1)_{\Omega}+\ell\left|\Omega_{1}\right| \\
& =\frac{\sigma}{R}\left(\kappa_{1}\left|\Omega_{1}\right|+\kappa_{2}\left|\Omega_{2}\right|\right)+\ell\left|\Omega_{1}\right|,
\end{aligned}
$$

where $\left|\Omega_{1}\right|=R^{n}|B|$ and $\left|\Omega_{2}\right|=|\Omega|-R^{n}|B|$, with $|B|$ the volume of the unit ball. A straightforward computation shows that the function $\phi$ has a unique minimum. It is attained at the point $R_{*}$, where $R_{*}$ is the unique solution of the equation

$$
\frac{\sigma(\kappa \mid 1)_{\Omega}}{R^{2}}=\left(\ell-\frac{\sigma[\kappa]}{R}\right)\left|S_{R}\right|,
$$

with $\left|S_{R}\right|=\left|S_{R}\left(x_{0}\right)\right|$ being the area of the sphere $S_{R}\left(x_{0}\right)$.

In the following we denote by $R_{*}$ the point where $\phi$ attains its (unique) minimum and by $R^{*}$ the largest number $R$ such that $\bar{B}_{R}\left(x_{0}\right) \subset \bar{\Omega}$, and we suppose that $R_{*}<R^{*}$. Then we have the following result.

Corollary 1.4. Let $c_{*}=\phi\left(R_{*}\right)$ be the minimum value of $\phi$ and let $c^{*}=\phi\left(R^{*}\right)$. Moreover, let $c_{0}:=E\left(u_{0}, \Gamma_{0}\right)$.

(a) If $c_{0}<c_{*}$, then problem (1.3) does not admit equilibrium states.

(b) If $c_{*}<c_{0}<c^{*}$ then (1.3) admits two branches of equilibrium states. The branch of equilibria $\left(\sigma / R, S_{R}\left(x_{0}\right)\right)$ with $0<R<R_{*}$ is linearly unstable, whereas the branch with $R_{*}<R<R^{*}$ is linearly stable.

(d) In case $c_{0}=c_{*}$ or $c_{0}>c^{*}$ the Stefan problem (1.3) admits one family of equilibrium states. All equilibria $\left(\sigma / R, S_{R}\left(x_{0}\right)\right)$ with $\phi(R)>c^{*}$ are linearly unstable.

(e) If $R_{0}:=[\kappa] \sigma / \ell>0$ and $\delta=0$, then the linearized problem is ill-posed for $R<R_{0}$.

Proof. This follows from Remark 1.2.(a), (1.12), and the fact that $\phi^{\prime}(R)$ is negative for $R<R_{*}$ and positive for $R_{*}<R<R^{*}$. 


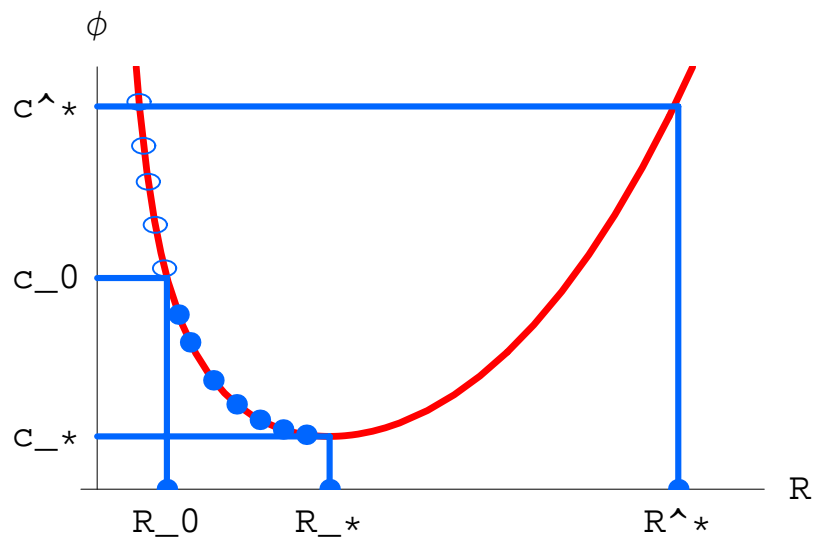

FiguRE 1. Stability diagram for $\kappa_{1}<\kappa_{2}$ and $\delta=0$; circled: illposed, dotted: unstable

Remarks 1.5. (a) We can show that $R_{*}$ is increasing with respect to $\sigma$ (and, for that matter, also with respect to $[\kappa])$. In order to see this, let $R_{*}(\sigma)$ denote the unique solution of (1.12). Then we have $R_{*}^{\prime}(\sigma)>0$. For this we note that (1.12) can be written as

$$
n \ell|B| R_{*}^{n+1}(\sigma)-\sigma\left(\kappa_{2}|\Omega|+(n-1)[\kappa]|B| R_{*}^{n}(\sigma)\right)=0 .
$$

Taking the derivative of this equation with respect to $\sigma$ yields

$$
\begin{aligned}
& n(n+1) \ell|B| R_{*}^{n-1}\left(R_{*}-\frac{(n-1)}{(n+1)} \frac{\sigma[\kappa]}{\ell}\right) R_{*}^{\prime}(\sigma) \\
& =\kappa_{2}|\Omega|+(n-1)[\kappa]|B| R_{*}^{n}=(\ell / \sigma) n|B| R_{*}^{n+1}(\sigma)>0 .
\end{aligned}
$$

Note that we have used (1.13) for the last equality . It remains to observe that the parenthesis in front of $R_{*}^{\prime}(\sigma)$ is always positive. This is clear in case $[\kappa] \leq 0$, and follows from the fact that $R_{*}$ is always greater than $R_{0}=\sigma[\kappa] / l$ in case $[\kappa]>0$.

We therefore see that increasing $\sigma$ increases $R_{*}(\sigma)$, showing that spheres with a fixed radius $R$ can lose stability as $\sigma$ increases.

(b) If one considers the case where the domain $\Omega_{1}$ is occupied by the solid phase, and $\Omega_{2}$ by the fluid phase, then the third and forth line in equation (1.3) need to be replaced by

$$
\begin{aligned}
& u_{i}=-\sigma H_{\Gamma}-\delta V \quad \text { on } \quad \Gamma(t) \\
& -\left[d \partial_{\nu} u\right]=(\ell+[\kappa] u) V \quad \text { on } \Gamma(t),
\end{aligned}
$$

and the energy functional by

$$
E(u(t), \Gamma(t)):=\int_{\Omega} \kappa u d x+\ell\left|\Omega_{2}(t)\right|=\int_{\Omega_{1}(t)} \kappa_{1} u_{1} d x+\int_{\Omega_{2}(t)} \kappa_{1} u_{2} d x+\ell\left|\Omega_{2}(t)\right|,
$$


while all other conventions are left unchanged. Thus formally one has to switch signs in the normal $\nu$ and in $\ell$ and $[\kappa]$. Then all the results and assertions stated in this paper remain valid for the equilibrium states $\left(-\sigma / R, S_{R}\left(x_{0}\right)\right)$.

The plan of this paper is the following. In Section 2 we will state a more general and concise version of Theorem 1.1. Its proof will be given in Sections 3-5. Finally, in Section 6 we will prove Theorem 1.3.

\section{MAIN THEOREM}

In this section we will introduce an appropriate functional analytic setting to study the eigenvalue problem (1.9). We always assume $l>0$ except when proving (f) of Theorem 1.1.

For the case $\delta=0$ we define the operator $L_{0}$ on $E_{0}:=L_{p}(\Omega) \times W_{p}^{2-2 / p}(\Sigma)$ by means of

$$
\begin{gathered}
D\left(L_{0}\right):=\left\{(v, \rho) \in W_{p}^{2}(\Omega \backslash \Sigma) \times W_{p}^{4-1 / p}(\Sigma):\left[d \partial_{\nu} v\right] \in W_{p}^{2-2 / p}(\Sigma),\right. \\
\left.\partial_{\nu} v=0 \text { on } \partial \Omega,[v]=0 \text { on } \Sigma, v=\sigma \mathcal{A}_{\Sigma} \rho \text { on } \Sigma\right\} \\
L_{0}(v, \rho):=\left((d / \kappa) \Delta v,\left[(d / l) \partial_{\nu} v\right]\right), \quad(v, \rho) \in D\left(L_{0}\right) .
\end{gathered}
$$

In case $\delta>0$ we set instead $E_{\delta}:=L_{p}(\Omega) \times W_{p}^{1-1 / p}(\Sigma)$ and we define $L_{\delta}$ by

$$
\begin{gathered}
D\left(L_{\delta}\right):=\left\{(v, \rho) \in W_{p}^{2}(\Omega \backslash \Sigma) \times W_{p}^{3-1 / p}(\Sigma):\right. \\
\left.\partial_{\nu} v=0 \text { on } \partial \Omega,[v]=0 \text { on } \Sigma, v-(\delta / l)\left[d \partial_{\nu} v\right]=\sigma \mathcal{A}_{\Sigma} \rho \text { on } \Sigma\right\}, \\
L_{\delta}(v, \rho):=\left((d / \kappa) \Delta v,\left[(d / l) \partial_{\nu} v\right]\right), \quad(v, \rho) \in D\left(L_{\delta}\right) .
\end{gathered}
$$

We remark that $L_{0}$ and $L_{\delta}$ only differ by their respective domains of definition. It will be shown in [7] that the operators $L_{\delta}$ generate an analytic semigroup on $E_{\delta}$. This property in conjunction with the spectral information contained in the next theorem will be crucial in proving global existence and convergence of solutions for problem (1.3) that start out close to an equilibrium, which will be provided in [15], see also Remark 1.2.(c).

Theorem 2.1. Suppose $1<p<\infty$ and let $l>0$. For $\delta \geq 0$ let $L_{\delta}$ be defined as above.

(a) The spectrum of $L_{\delta}$ consists of countably many real eigenvalues of finite algebraic multiplicity, and it is independent of $p$.

(b) 0 is an eigenvalue of $L_{\delta}$ with geometric multiplicity $(n+1)$. The null space of $L_{\delta}$ is spanned by

$$
\left(-1, Y_{0}\right),\left(0, Y_{1}\right), \ldots,\left(0, Y_{n}\right),
$$

where $Y_{0}=R^{2} / \sigma$, and where $Y_{j}, 1 \leq j \leq n$, are the spherical harmonics of degree 1 (normalized by the orthogonality condition $\left(Y_{i} \mid Y_{j}\right)_{\Sigma}=\delta_{i j}$ ). 
(c) Suppose that the degeneracy condition

$$
(\kappa \mid 1)_{\Omega}:=\kappa_{1}\left|\Omega_{1}\right|+\kappa_{2}\left|\Omega_{2}\right|=l|\Sigma| R^{2} / \sigma
$$

holds. Then the eigenvalue 0 has algebraic multiplicity $(n+2)$.

(d) If the degeneracy condition (2.2) does not hold, then 0 is semi-simple, that is, $N\left(L_{\delta}^{2}\right)=N\left(L_{\delta}\right)$.

(e) If $\sigma(\kappa \mid 1)_{\Omega} \leq l|\Sigma| R^{2}$, then $L_{\delta}$ has no positive eigenvalues.

(f) If $\sigma(\kappa \mid 1)_{\Omega}>l|\Sigma| R^{2}$, then $L_{\delta}$ has exactly one positive simple eigenvalue.

Proof. (a) By the compact embeddings $D\left(L_{\delta}\right) \hookrightarrow E_{\delta}$, the spectrum of $L_{\delta}$ consists of eigenvalues of finite algebraic multiplicity. The assertion that all eigenvalues are real will be proved in Section 4 .

Let $1<p<\infty$ be fixed and suppose that $\lambda$ is an eigenvalue of $L_{\delta}$ with corresponding eigenfunction $(v, \rho)$. Then $v \in W_{p}^{2}(\Omega \backslash \Sigma)$ and $v$ solves the elliptic transmission problem



with $\rho \in W_{p}^{4-\operatorname{sign}(\delta)-1 / p}(\Sigma)$, where $\operatorname{sign}(\delta)=1$ if $\delta>0$ and $\operatorname{sign}(\delta)=0$ if $\delta=0$. Due to Sobolev's imbedding theorem we have that $\rho \in W_{p_{1}}^{1-1 / p_{1}}(\Sigma)$, where $p_{1} \in$ $(p, \infty)$ is appropriately chosen. Proposition 5.1 then yields $v \in W_{p_{1}}^{2}(\Omega \backslash \Sigma)$. Next we recall that $\rho$ satisfies the equation

$$
\sigma \mathcal{A}_{\Sigma} \rho=v-(\delta / l)\left[d \partial_{\nu} v\right]=: h \quad \text { on } \quad \Sigma .
$$

Since $v \in W_{p_{1}}^{2}(\Omega \backslash \Sigma)$ we see that $h \in W_{p_{1}}^{2-\operatorname{sign}(\delta)-1 / p_{1}}(\Sigma)$, and we obtain from the properties of the elliptic differential operator $\mathcal{A}_{\Sigma}$ that $\rho \in W_{p_{1}}^{4-\operatorname{sign}(\delta)-1 / p_{1}}(\Sigma)$. The arguments given above can now be reiterated a finite number of times to show that

$$
(v, \rho) \in W_{q}^{2}(\Omega \backslash \Sigma) \times W_{q}^{4-\operatorname{sign}(\delta)-1 / q}(\Sigma)
$$

for any fixed $q>p$. Clearly, this is also true for any $q<p$. We have, thus, shown that the spectrum of $L_{\delta}$ is independent of $p$. The properties listed in (b)-(d) are proved in Section 3, assertion (e) is shown in Section 4 while (f) is established in Section 5 .

Proposition 2.2. Let $1<p<\infty$. Suppose that $(\lambda, v, \rho) \in \mathbb{R} \times W_{p}^{2}(\Omega \backslash \Sigma) \times W_{p}^{2}(\Sigma)$ solves the eigenvalue problem (1.9). Then the functions $(v, \rho)$ are smooth, that is,

$$
\left.v\right|_{\Omega_{i}} \in C^{\infty}\left(\bar{\Omega}_{i}\right), \quad \rho \in C^{\infty}(\Sigma) .
$$

Proof. This follows from a similar bootstrapping argument as in the proof of Theorem 2.1.(a), based on regularity properties of the the elliptic transmission problems (3.4) and (5.2), and regularity properties of the differential operator $\mathcal{A}_{\Sigma}$. 
Due to Theorem 2.1 and Proposition 2.2 we may restrict our attention to the eigenvalue problem (1.9) in the Hilbert space setting of $L_{2}(\Omega) \times L_{2}(\Sigma)$. In the following we use the notation $(\cdot \mid \cdot)_{\Omega}$ and $\|\cdot\|_{\Omega}$ for the inner product and the norm in $L_{2}(\Omega)$, respectively, and similarly for $L_{2}(\Sigma)$.

\section{The Trivial Eigenvalue}

Let us first look at the eigenvalue problem (1.9) with $\lambda=0$. Obviously, here $l \in \mathbb{R}$ can be arbirtary, and also $\delta \in \mathbb{R}$. For this purpose we recall some properties of the operator $\mathcal{A}_{\Sigma}$.

Proposition 3.1. Let $\Sigma=S_{R}\left(x_{0}\right) \subset \mathbb{R}^{n}$ be a sphere of radius $R$ and center $x_{0}$, and let

$$
A_{\Sigma}=-\frac{1}{n-1}\left(\frac{n-1}{R^{2}}+\Delta_{\Sigma}\right)
$$

be defined on $L_{2}(\Sigma)$ with domain $W_{2}^{2}(\Sigma)$. Then

(a) $A_{\Sigma}$ is self-adjoint.

(b) The kernel of $A_{\Sigma}$ is given by $N\left(A_{\Sigma}\right)=\operatorname{span}\left\{Y_{1}, \ldots, Y_{n}\right\}$, where $Y_{j}$ denote the sperical harmonics of degree 1 on $\Sigma$, normalized by $\left(Y_{i} \mid Y_{j}\right)_{\Sigma}=\delta_{i j}$.

(c) The range of $A_{\Sigma}, R\left(A_{\Sigma}\right)$, is closed, and we have $L_{2}(\Sigma)=N\left(A_{\Sigma}\right) \oplus R\left(A_{\Sigma}\right)$.

(d) There is precisely one negative eigenvalue, namely $-1 / R^{2}$, with eigenfunction 1, which is simple.

(e) $A_{\Sigma}$ is positive semi-definite on $L_{2,0}(\Sigma)=\left\{\rho \in L_{2}(\Sigma):(\rho \mid 1)_{\Sigma}=0\right\}$ and positive definite on

$$
L_{2,0}(\Sigma) \cap R\left(A_{\Sigma}\right)=\left\{\rho \in L_{2}(\Sigma):(\rho \mid 1)_{\Sigma}=\left(\rho \mid Y_{j}\right)_{\Sigma}=0, j=1, \ldots, n\right\} .
$$

Proof. We can assume without loss of generality that $\Sigma=S_{R}(0)=R \mathbb{S}^{n-1}$, where $\mathbb{S}^{n-1}$ denotes the standard unit sphere in $\mathbb{R}^{n}$. Let $\Phi: \Sigma \rightarrow \mathbb{S}^{n-1}$ be defined by $p \mapsto(1 / R) p$. Then $\Phi$ is a smooth diffeomorphism of $\Sigma$ into $\mathbb{S}^{n-1}$ and one readily verifies that

$$
(g \mid h)_{L_{2}(\Sigma)}=R^{n-1}\left(\Phi_{*} g \mid \Phi_{*} h\right)_{L_{2}\left(\mathbb{S}^{n-1}\right)}, \quad \Delta_{\Sigma}=\left(1 / R^{2}\right) \Phi^{*} \Delta_{\mathbb{S}^{n-1}} \Phi_{*}
$$

where $\Phi^{*}$ and $\Phi_{*}$ are the pull-back and push-forward operators, respectively. We then have

$$
\left(\lambda-A_{\Sigma}\right) \rho=0 \Longleftrightarrow\left(\lambda+\frac{1}{(n-1) R^{2}}\left((n-1)+\Delta_{\mathbb{S} n-1}\right)\right) \Phi_{*} \rho=0
$$

and this shows that $\lambda$ is an eigenvalue of $A_{\Sigma}$ iff

$$
\lambda=\frac{1}{(n-1) R^{2}}(\mu-(n-1))
$$

with $\mu$ an eigenvalue of $-\Delta_{\mathbb{S}^{n-1}}$. The assertions in (a)-(b) and (d)-(e) follow now from (3.1)-(3.3) and well-known results for the Laplace-Beltrami operator on $\mathbb{S}^{n-1}$, see for instance [30, Section 31]. Since $A_{\Sigma}$ has compact resolvent we conclude that 
$R\left(A_{\Sigma}\right)$ is closed, and the fact that $A_{\Sigma}$ is self-adjoint then implies the remaining assertion in (c).

Before we proceed we need the following result on the elliptic transmission problem



Proposition 3.2. Let $1<p<\infty$. Then

(a) The transmission problem (3.4) has a solution $v \in W_{p}^{2}(\Omega \backslash \Sigma)$ if and only if $(f, g) \in L_{p}(\Omega) \times W_{p}^{1-1 / p}(\Sigma)$ and the compatibility condition

$$
(f \mid 1)_{\Omega}+(g \mid 1)_{\Sigma}=0
$$

is satisfied. The solution is unique with the normalization $(\kappa \mid v)_{\Omega}=0$.

(b) Let $v=T_{0} g$ be the unique solution of (3.4) with $f=0,(g \mid 1)_{\Sigma}=0$ and $(\kappa \mid v)_{\Omega}=0$. Then $T_{0}$ is self-adjoint and positive definite on $L_{2}(\Sigma)$, that is, there exists a positive constant $c=c\left(d_{i}, \Omega_{i}\right)$ such that

$$
\left(T_{0} g \mid g\right)_{L_{2}(\Sigma)} \geq c\|g\|_{L_{2}(\Sigma)}^{2}, \quad g \in W_{2}^{1 / 2}(\Sigma) .
$$

Proof. (a) follows from known results in elliptic theory since the LopatinskiiShapiro conditions are satisfied.

(b) Let $g, h \in W_{2}^{1 / 2}(\Sigma)$ be given. Then we have

$$
\begin{aligned}
\left(T_{0} g \mid h\right)_{\Sigma}=\left(T_{0} g \mid\left[-d \partial_{\nu} T_{0} h\right]\right)_{\Sigma} & =\left(d \nabla T_{0} g \mid \nabla T_{0} h\right)_{\Omega} \\
& =\left(-\left[d \partial_{\nu} T_{0} g\right] \mid T_{0} h\right)_{\Sigma}=\left(g \mid T_{0} h\right)_{\Sigma},
\end{aligned}
$$

thus showing that $T_{0}$ is symmetric. For $v:=T_{0} g$ the computation above yields

$$
\left(T_{0} g \mid g\right)_{\Sigma}=(d \nabla v \mid \nabla v)_{\Omega}
$$

On the other hand, setting $v_{i}=\left.v\right|_{\Omega_{i}}$ we obtain

$$
\begin{aligned}
\|g\|_{L_{2}(\Sigma)} & =\left\|d_{1} \partial_{\nu} v_{1}-d_{2} \partial_{\nu} v_{2}\right\|_{L_{2}(\Sigma)} \leq c\left(\left\|v_{1}\right\|_{W_{2}^{2}\left(\Omega_{1}\right)}+\left\|v_{2}\right\|_{W_{2}^{2}\left(\Omega_{2}\right)}\right) \\
& \leq c\left(\left\|v_{1}\right\|_{L_{2}\left(\Omega_{1}\right)}+\left\|\Delta v_{1}\right\|_{L_{2}\left(\Omega_{1}\right)}+\left\|v_{2}\right\|_{L_{2}\left(\Omega_{2}\right)}+\left\|\Delta v_{2}\right\|_{L_{2}\left(\Omega_{2}\right)}\right) \\
& =c\|v\|_{L_{2}(\Omega)} \leq c\|v\|_{W_{2}^{1}(\Omega)} \leq c\|\nabla v\|_{L_{2}(\Omega)} \leq c\left(T_{0} g \mid g\right)_{\Sigma}^{1 / 2} .
\end{aligned}
$$

Here we used the fact that

$$
v=T_{0} g \in W_{2}^{1}(\Omega) \cap W_{2}^{2}(\Omega \backslash \Sigma) .
$$

Moroever, we used that $\left(\|\cdot\|_{L_{2}\left(\Omega_{i}\right)}+\|\Delta \cdot\|_{L_{2}\left(\Omega_{i}\right)}\right)$ defines an equivalent norm on $W_{2}^{2}\left(\Omega_{i}\right)$, and also that $\|\nabla u\|_{L_{2}(\Omega)}$ defines an equivalent norm on $W_{2}^{1}(\Omega)$ for all functions $u \in W_{2}^{1}(\Omega)$ with $(\kappa \mid u)_{\Omega}=0$. This completes the proof of Proposition 4.2. 
We are now ready to establish the assertions (b)-(d) of Theorem 2.1.

(b) Suppose that $(v, \rho)$ is a solution of (1.9) with $\lambda=0$. Then taking the inner product of $(1.9)_{1}$ with $v$, the divergence theorem and (1.9) $)_{2,4}$ show that $v$ is constant on $\Omega \backslash \Sigma$, hence $v$ is constant on $\Omega$ and $v=\sigma \mathcal{A}_{\Sigma} \rho$ due to (1.9) ${ }_{3}$. A special solution of this problem is $\rho_{0}=-R^{2} v / \sigma$, and the solutions of the corresponding homogeneous equation are the spherical harmonics $Y_{j}$ on $\Sigma$ for $j=1, \ldots, n$. Thus we obtain an $(n+1)$-dimensional null space spanned by $(2.1)$, and this proves Theorem 2.1(b).

This null space is tangent to the $(\mathrm{n}+1)$-dimensional manifold of equilibria, where $\left(0, Y_{j}\right)$ corrrespond to the center $x_{0}$, and $\left(-1, Y_{0}\right)$ to the radius $R$. Note that the null spaces of $L_{0}$ and $L_{\delta}, \delta>0$, coincide.

(c) Suppose that $(2.2)$ holds. Then there exists a pair $\left(v^{*}, \rho^{*}\right) \in N\left(L_{\delta}^{2}\right) \backslash N\left(L_{\delta}\right)$. Indeed, this can be seen as follows: we first solve (3.4) with $(f, g)=\left(-\kappa, l R^{2} / \sigma\right)$. According to Proposition 3.2, this problem has a unique solution $v_{0}$ with $\left(\kappa \mid v_{0}\right)_{\Omega}=$ 0 since the necessary compatibility condition is precisely (2.2).

Set $v^{*}=v_{0}+l \sum_{j=1}^{n} \alpha_{j} T_{0} Y_{j}$. Then $v^{*}$ satisfies

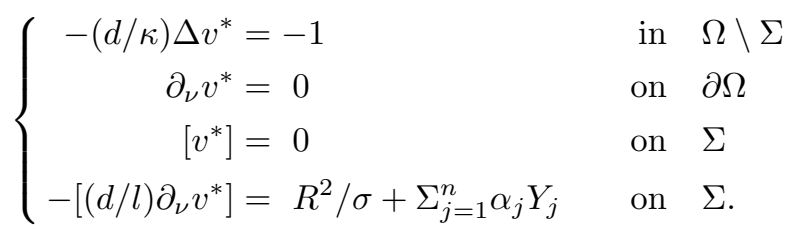

We now want to solve $v^{*}=\sigma A_{\Sigma} \rho+(\delta / l)\left[d \partial_{\nu} v^{*}\right]$ in terms of $\rho$, that is, we consider the problem

$$
\sigma A_{\Sigma} \rho=v^{*}-(\delta / l)\left[d \partial_{\nu} v^{*}\right]=: h .
$$

According to Proposition 4.1(b)-(c) this problem has a solution $\rho^{*}$ if and only if $\left(h \mid Y_{i}\right)_{\Sigma}=0$ for $i=1, \ldots, n$.

The conditions $\left(h \mid Y_{i}\right)_{\Sigma}=0$ will be employed to determine the coefficients $\alpha_{j}$. A short computation yields

$$
\left(h \mid Y_{i}\right)_{\Sigma}=0 \Leftrightarrow \delta \alpha_{i}+\sum_{j=1}^{n} l\left(T_{0} Y_{j} \mid Y_{i}\right)_{\Sigma} \alpha_{j}=-\left(v_{0} \mid Y_{i}\right)_{\Sigma}, \quad i=1, \ldots, n .
$$

Since $T_{0}$ is self-adjoint and positive definite on $L_{2}(\Sigma)$ there exists a unique solution of this system, see also (6.7). It is easy to see that $\left(v^{*}, \rho^{*}\right) \in D\left(L_{\delta}^{2}\right)$, that $L_{\delta}\left(v^{*}, \rho^{*}\right) \neq(0,0)$, and that $L_{\delta}^{2}\left(v^{*}, \rho^{*}\right)=(0,0)$. This facts in combination with part (b) show that

$$
N\left(L_{\delta}^{2}\right)=N\left(L_{\delta}\right) \oplus \operatorname{span}\left\{\left(v^{*}, \rho^{*}\right)\right\},
$$

in the degenerate case where (2.2) holds. 
Next we show, still in the degenerate case $(2.2)$, that $N\left(L_{\delta}^{3}\right)=N\left(L_{\delta}^{2}\right)$. In fact, if $(v, \rho) \in N\left(L_{\delta}^{3}\right)$ then $L_{\delta}(v, \rho)=\left(v_{N}, \rho_{N}\right)+\beta\left(v^{*}, \rho^{*}\right)$ for some $\left(v_{N}, \rho_{N}\right) \in N\left(L_{\delta}\right)$ and some scalar $\beta$. For solvability of this equation, the compatibility condition

$$
\left(\kappa\left(v_{N}+\beta v^{*}\right) \mid 1\right)_{\Omega}+l\left(\rho_{N}+\beta \rho^{*} \mid 1\right)_{\Sigma}=0
$$

must be valid. Due to the degeneracy condition we have $\left(\kappa v_{N} \mid 1\right)_{\Omega}+l\left(\rho_{N} \mid 1\right)_{\Sigma}=0$, and the compatibility condition is reduced to $\left.\beta\left\{\left(\kappa v^{*}\right) \mid 1\right)_{\Omega}+l\left(\rho^{*} \mid 1\right)_{\Sigma}\right\}=0$. Using the property that $-(d / \kappa) \Delta v^{*}=-1$, see equation (3.5), we obtain

$$
\begin{aligned}
& -\left\{\left(\kappa v^{*} \mid 1\right)_{\Omega}+l\left(\rho^{*} \mid 1\right)_{\Sigma}\right\}=-\left(d \Delta v^{*} \mid v^{*}\right)_{\Omega}-l\left(\rho^{*} \mid 1\right)_{\Sigma} \\
& \quad=\left\|\sqrt{d} \nabla v^{*}\right\|_{\Omega}^{2}+\left(\left[d \partial_{\nu} v^{*}\right] \mid v^{*}\right)_{\Sigma}-l\left(\rho^{*} \mid 1\right)_{\Sigma} \\
& =\left\|\sqrt{d} \nabla v^{*}\right\|_{\Omega}^{2}-l\left(R^{2} / \sigma+\sum_{j=1}^{n} \alpha_{j} Y_{j} \mid \sigma A_{\Sigma} \rho^{*}\right)_{\Sigma}+(\delta / l)\left\|\left[d \partial_{\nu} v^{*}\right]\right\|_{\Sigma}^{2}-l\left(\rho^{*} \mid 1\right)_{\Sigma} \\
& \quad=\left\|\sqrt{d} \nabla v^{*}\right\|_{\Omega}^{2}+(\delta / l)\left\|\left[d \partial_{\nu} v^{*}\right]\right\|_{\Sigma}^{2}
\end{aligned}
$$

since $A_{\Sigma}$ is selfadjoint on $L_{2}(\Sigma)$ and $\sigma A_{\Sigma}\left(R^{2} / \sigma+\sum_{j=1}^{n} \alpha_{j} Y_{j}\right)=-1$, see Proposition 4.1. This implies $\beta=0$, i.e. $(v, \rho) \in N\left(L_{\delta}^{2}\right)$, thus establishing Theorem 2.1(c).

(d) Let us examine when the eigenvalue $\lambda_{0}=0$ of $L_{\delta}$ is semi-simple. Assume that $(v, \rho) \in D\left(L_{\delta}^{2}\right)$ is such that $L_{\delta}^{2}(v, \rho)=0$. Then

$$
L_{\delta}(v, \rho)=\alpha_{0}\left(-1, Y_{0}\right)+\sum_{j=1}^{n} \alpha_{j}\left(0, Y_{j}\right)
$$

This implies that

$$
\left\{\begin{aligned}
-(d / \kappa) \Delta v & =-\alpha_{0} & & \text { in } \quad \Omega \backslash \Sigma \\
\partial_{\nu} v & =0 & & \text { on } \quad \partial \Omega \\
{[v] } & =0 & & \text { on } \Sigma \\
-\left[(d / l) \partial_{\nu} v\right] & =\sum_{j=0}^{n} \alpha_{j} Y_{j} & & \text { on } \Sigma \\
v & =\sigma \mathcal{A}_{\Sigma} \rho+(\delta / l)\left[d \partial_{\nu} v\right] & & \text { on } \Sigma .
\end{aligned}\right.
$$

According to Proposition 3.2 we necessarily have

$$
\alpha_{0}\left(\kappa_{1}\left|\Omega_{1}\right|+\kappa_{2}\left|\Omega_{2}\right|\right)=l \sum_{j=0}^{n} \alpha_{j}\left(Y_{j} \mid 1\right)_{\Sigma}=l \alpha_{0}|\Sigma| R^{2} / \sigma
$$

since the mean value of $Y_{j}$ over $\Sigma$ is zero for $j \geq 1$. Assuming the non-degeneracy condition

$$
(\kappa \mid 1)_{\Omega}:=\kappa_{1}\left|\Omega_{1}\right|+\kappa_{2}\left|\Omega_{2}\right| \neq l|\Sigma| R^{2} / \sigma
$$

we conclude that $\alpha_{0}=0$. But then

$$
0=-\int_{\Omega} d \Delta v v d x=\int_{\Omega} d|\nabla v|^{2} d x+\int_{\Sigma}\left[d \partial_{\nu} v\right] v d s
$$


which further yields

$0=\|\sqrt{d} \nabla v\|_{\Omega}^{2}-l \sigma \sum_{j=1}^{n} \alpha_{j}\left(Y_{j} \mid \mathcal{A}_{\Sigma} \rho\right)_{\Sigma}+(\delta / l)\left\|\left[d \partial_{\nu} v\right]\right\|_{\Sigma}^{2}=\|\sqrt{d} \nabla v\|_{\Omega}^{2}+(\delta / l)\left\|\left[d \partial_{\nu} v\right]\right\|_{\Sigma}^{2}$

since $\mathcal{A}_{\Sigma}$ is selfadjoint on $L_{2}(\Sigma)$ and $A_{\Sigma} Y_{j}=0$ for $j \geq 1$. We conclude that $v$ is constant in $\Omega$ and that $0=-\left[d \partial_{\nu} v\right]=l \sum_{j=1}^{n} \alpha_{j} Y_{j}$, hence $\alpha_{j}=0$ for all $j$. This shows that $\lambda_{0}=0$ is a semi-simple eigenvalue of $L_{\delta}$, that is, $N\left(L_{\delta}^{2}\right)=N\left(L_{\delta}\right)$ for $\delta \geq 0$, provided the non-degeneracy condition (3.7) is valid, and this proves the assertion of Theorem 2.1(d).

\section{Nontrivial Eigenvalues}

Now we consider the eigenvalue problem (1.9) for $\lambda \in \mathbb{C}, \lambda \neq 0$, in case $l>0$. Suppose that $\lambda \neq 0$ is an eigenvalue with nontrivial eigenfunction $(v, \rho)$. Taking the inner product in $L_{2}(\Omega)$ of the first equation in (1.9) with $v$ and using the divergence theorem we get

$$
\begin{aligned}
\lambda\|\sqrt{\kappa} v\|_{\Omega}^{2}=(d \Delta v \mid v)_{\Omega} & =-\|\sqrt{d} \nabla v\|_{\Omega}^{2}-\left(\left[d \partial_{\nu} v\right] \mid v\right)_{\Sigma} \\
& =-\|\sqrt{d} \nabla v\|_{\Omega}^{2}-(\delta / l)\left\|\left[d \partial_{\nu} v\right]\right\|_{\Sigma}^{2}-\lambda l \sigma\left(\rho \mid A_{\Sigma} \rho\right)_{\Sigma},
\end{aligned}
$$

hence we obtain the identity

$$
\lambda\left(\|\sqrt{\kappa} v\|_{\Omega}^{2}+l \sigma\left(\rho \mid A_{\Sigma} \rho\right)_{\Sigma}\right)+\|\sqrt{d} \nabla v\|_{\Omega}^{2}+(\delta / l)\left\|\left[d \partial_{\nu} v\right]\right\|_{\Sigma}^{2}=0 .
$$

If $\operatorname{Im} \lambda \neq 0$ then $\|\sqrt{\kappa} v\|_{\Omega}^{2}+l \sigma\left(\rho \mid A_{\Sigma} \rho\right)_{\Sigma}=0$, hence $\|\sqrt{d} \nabla v\|_{\Omega}^{2}+(\delta / l)\left\|\left[d \partial_{\nu}\right]\right\|_{\Sigma}^{2}=0$. We conclude that $v$ is constant and $(1.9)_{1,4}$ now implies that $(v, \rho)=(0,0)$ since $\lambda \neq 0$. Therefore the eigenvalues and eigenfunctions are real, thus establishing Theorem 2.1(a).

Using $\lambda l \rho=\left[d \partial_{\nu} v\right]$ and the fact that $\lambda$ is real we may rewrite (4.1) as

$$
\lambda\left(\|\sqrt{\kappa} v\|_{\Omega}^{2}+l \sigma\left(\rho \mid A_{\Sigma} \rho\right)_{\Sigma}+\lambda l \delta\|\rho\|_{\Sigma}^{2}\right)+\|\sqrt{d} \nabla v\|_{\Omega}^{2}=0 .
$$

Integrating the eigenvalue equation (1.9) we obtain

$$
\lambda(v \mid \kappa)_{\Omega}=(d \Delta v \mid 1)_{\Omega}=-\left(\left[d \partial_{\nu} v\right] \mid 1\right)_{\Sigma}=-\lambda l(\rho \mid 1)_{\Sigma},
$$

hence dividing by $\lambda$,

$$
(v \mid \kappa)_{\Omega}+l(\rho \mid 1)_{\Sigma}=0 .
$$

Splitting $\rho=\rho_{0}+\bar{\rho}$ and $v=v_{0}+\bar{v}$, where $\left(\rho_{0} \mid 1\right)_{\Sigma}=\left(v_{0} \mid \kappa\right)_{\Omega}=0$, from (4.2) and (4.3) we derive an identity equivalent to (4.2), namely

$$
\begin{aligned}
\lambda\left(\left\|\sqrt{\kappa} v_{0}\right\|_{\Omega}^{2}+l \sigma\left(\rho_{0} \mid A_{\Sigma} \rho_{0}\right)_{\Sigma}\right. & \left.+\lambda l \delta\left\|\rho_{0}\right\|_{\Sigma}^{2}\right)+\|\sqrt{d} \nabla v\|_{\Omega}^{2} \\
& +\lambda\left(\lambda \delta+\frac{l|\Sigma|}{(\kappa \mid 1)_{\Omega}}-\frac{\sigma}{R^{2}}\right) l|\Sigma| \bar{\rho}^{2}=0 .
\end{aligned}
$$


Since $A_{\Sigma}$ is positive semidefinite on $L_{2,0}(\Sigma)$, the $L_{2}$-functions with mean zero, we see that in case $\lambda>0,(4.4)$ implies $v=$ constant and hence $(v, \rho)=(0,0)$, provided

$$
(\kappa \mid 1)_{\Omega} \leq l|\Sigma| R^{2} / \sigma .
$$

Consequently, (1.9) cannot have positive eigenvalues if the stability condition (4.5) is satisfied, thus proving the assertion of Theorem 2.1(e).

\section{The Unstable Eigenvalue}

So far we know that for $l>0$ and

$$
\zeta:=\frac{\sigma(\kappa \mid 1)_{\Omega}}{l|\Sigma| R^{2}} \leq 1
$$

there are no positive eigenvalues, however, the algebraic eigenspace of $L_{\delta}$ rises in dimension by one when $\zeta$ becomes one. This indicates that for $\zeta>1$ there is exactly one algebraically simple eigenvalue $\lambda_{*}>0$. We want to prove that this is indeed the case. In order to do so, we consider the following transmission problem

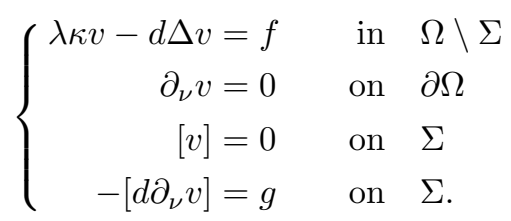

Then the following result holds.

Proposition 5.1. Let $1<p<\infty$ and $\operatorname{Re} \lambda>0$. Then

(a) Problem (5.2) has precisely one solution $v \in W_{p}^{1}(\Omega) \cap W_{p}^{2}(\Omega \backslash \Sigma)$ if and only if $(f, g) \in L_{p}(\Omega) \times W_{p}^{1-1 / p}(\Sigma)$.

(b) Let $T_{\lambda}$ be the solution operator for (5.2) with $f=0$. Given any number $\theta \in(0, \pi)$ there exists positive numbers $\lambda_{0}=\lambda_{0}\left(\theta, d_{i}, \kappa_{i}, \Omega_{i}\right)$ and $M_{0}=$ $M_{0}\left(\theta, d_{i}, \kappa_{i}, \Omega_{i}\right)$ such that

$$
\left\|T_{\lambda} g\right\|_{L_{p}(\Sigma)} \leq M_{0}|\lambda|^{-1 / 2}\|g\|_{L_{p}(\Sigma)}
$$

for $g \in W_{p}^{1-1 / p}(\Sigma)$, whenever $|\lambda| \geq \lambda_{0}$ and $|\arg \lambda| \leq \theta$.

(c) For $\lambda>0, T_{\lambda}$ is positive definite on $L_{2}(\Sigma)$, that is, there exists a positive constant $\beta=\beta\left(d_{i}, \kappa_{i}, \Omega_{i}\right)$ such that

$$
\left(T_{\lambda} g \mid g\right)_{L_{2}(\Sigma)} \geq \beta \frac{\sqrt{\lambda}}{1+\lambda}\|g\|_{L_{2}(\Sigma)}^{2}, \quad g \in W_{2}^{1 / 2}(\Sigma) .
$$

Proof. (a) follows from known results in elliptic theory.

(b) Suppose that $\Omega_{1}=\mathbb{R}_{-}^{n}$ and $\Omega_{2}=\mathbb{R}_{+}^{n}$, with $\mathbb{R}_{ \pm}^{n}=\left\{\left(x^{\prime}, x_{n}\right) \in \mathbb{R}^{n}: \pm x_{n}<0\right\}$. Then one readily obtains that

$$
\left.T_{\lambda} g\right|_{\mathbb{R}^{n-1} \times\{0\}}=\mathcal{F}^{-1}\left(m_{\lambda} \mathcal{F} g\right),
$$


where $\mathcal{F}$ denotes the Fourier transform in the tangential variables and where

$$
m_{\lambda}(\xi)=\frac{1}{\sqrt{d_{1}} \sqrt{\kappa_{1} \lambda+d_{1}|\xi|^{2}}+\sqrt{d_{2}} \sqrt{\kappa_{2} \lambda+d_{2}|\xi|^{2}}} .
$$

The assertion then follows from Mikhlin's multiplier theorem. The general case can be obtained by the usual procedure of localization.

(c) Let $g, h \in W_{2}^{1 / 2}(\Sigma)$ be given. Then we have

$$
\begin{aligned}
\left(T_{\lambda} g \mid h\right)_{\Sigma}=\left(T_{\lambda} g \mid\left[-d \partial_{\nu} T_{\bar{\lambda}} h\right]\right)_{\Sigma} & =\lambda\left(\kappa T_{\lambda} g \mid T_{\bar{\lambda}} h\right)_{\Omega}+\left(d \nabla T_{\lambda} g \mid \nabla T_{\bar{\lambda}} h\right)_{\Omega} \\
& =\left(-\left[d \partial_{\nu} T_{\lambda} g\right] \mid T_{\bar{\lambda}} h\right)_{\Sigma}=\left(g \mid T_{\bar{\lambda}} h\right)_{\Sigma},
\end{aligned}
$$

showing that $T_{\lambda}^{*}=T_{\bar{\lambda}}$, in particular $T_{\lambda}$ is symmetric for $\lambda>0$. For $v:=T_{\lambda} g$, $\lambda>0$, the computation above yields

$$
\left(T_{\lambda} g \mid g\right)_{\Sigma}=\lambda(\kappa v \mid v)_{\Omega}+(d \nabla v \mid \nabla v)_{\Omega} .
$$

Setting $v_{i}=\left.v\right|_{\Omega_{i}}$ we conclude similarly as in the proof of Proposition 3.2 that

$$
\begin{aligned}
\|g\|_{L_{2}(\Sigma)} & =\left\|d_{1} \partial_{\nu} v_{1}-d_{2} \partial_{\nu} v_{2}\right\|_{L_{2}(\Sigma)} \leq c\left(\left\|d_{1} v_{1}\right\|_{W_{2}^{2}\left(\Omega_{1}\right)}+\left\|d_{2} v_{2}\right\|_{W_{2}^{2}\left(\Omega_{2}\right)}\right) \\
& \leq c\left(\left\|d_{1} v_{1}\right\|_{L_{2}\left(\Omega_{1}\right)}+\left\|d_{1} \Delta v_{1}\right\|_{L_{2}\left(\Omega_{1}\right)}+\left\|d_{2} v_{2}\right\|_{L_{2}\left(\Omega_{2}\right)}+\left\|d_{2} \Delta v_{2}\right\|_{L_{2}\left(\Omega_{2}\right)}\right) \\
& =c\left(\left\|d_{1} v_{1}\right\|_{L_{2}\left(\Omega_{1}\right)}+\lambda\left\|\kappa_{1} v_{1}\right\|_{L_{2}\left(\Omega_{1}\right)}+\left\|d_{2} v_{2}\right\|_{L_{2}\left(\Omega_{2}\right)}+\lambda\left\|\kappa_{2} v_{2}\right\|_{L_{2}\left(\Omega_{2}\right)}\right) \\
& \left.\leq c_{\lambda} \sqrt{\lambda}\|v\|_{L_{2}(\Omega)} \leq c_{\lambda}\left(\sqrt{\lambda}\|v\|_{L_{2}(\Omega)}+\|\nabla v\|_{L_{2}(\Omega)}\right) \leq c_{\lambda}\left(T_{\lambda} g \mid g\right)_{\Sigma}\right)^{1 / 2},
\end{aligned}
$$

where $c_{\lambda}=c\left(d_{i}, \kappa_{i}, \Omega_{i}\right)(1+\lambda) / \sqrt{\lambda}$. In the estimates above we have used that $v=T_{\lambda} g \in W_{2}^{1}(\Omega) \cap W_{2}^{2}(\Omega \backslash \Sigma)$, that $\left(\|\cdot\|_{L_{2}\left(\Omega_{i}\right)}+\|\Delta \cdot\|_{L_{2}\left(\Omega_{i}\right)}\right)$ defines an equivalent norm on $W_{2}^{2}\left(\Omega_{i}\right)$ and, lastly, we employed (5.3). This completes the proof of Proposition 5.1.

We assume now that $\lambda>0$ is a fixed number. For $\rho \in W_{2}^{1 / 2}(\Sigma)$ given let $v$ be the solution of the transmission problem



Then $v=T_{\lambda}(-\lambda l \rho)=-\lambda l T_{\lambda} \rho$ with $T_{\lambda}$ the solution operator introduced above. Inserting this representation of $v$ into the equation $v=\sigma A_{\Sigma} \rho+\lambda \delta \rho$ we obtain the problem

$$
\lambda \delta \rho+\lambda l T_{\lambda} \rho+\sigma A_{\Sigma} \rho=0
$$

which is equivalent to the eigenvalue problem.

Setting $B_{\lambda}(s):=\lambda \delta I+\lambda l T_{\lambda}+s \sigma A_{\Sigma}$ for $s>0$ and employing Proposition 5.1.(c) we obtain the estimate

$$
\begin{aligned}
\left(B_{\lambda}(s) \rho \mid \rho\right)_{\Sigma} & \geq \lambda(\delta+\gamma l)\|\rho\|_{\Sigma}^{2}+s \sigma\left(A_{\Sigma} \rho \mid \rho\right)_{\Sigma} \\
& =\lambda(\delta+\gamma l)\left\|\rho_{0}\right\|_{\Sigma}^{2}+s \sigma\left(A_{\Sigma} \rho_{0} \mid \rho_{0}\right)_{\Sigma}+\left\{\lambda(\delta+\gamma l)-s \sigma / R^{2}\right\}|\Sigma| \bar{\rho}^{2},
\end{aligned}
$$


where $\gamma=\beta \sqrt{\lambda} /(1+\lambda)$ and $\rho=\rho_{0}+\bar{\rho}$ with $\left(\rho_{0} \mid 1\right)_{\Sigma}=0$. Since $\left(A_{\Sigma} \rho_{0} \mid \rho_{0}\right)_{\Sigma} \geq 0$ we see that all terms in the previous line are nonnegative, provided $\lambda(\delta+\gamma l) \geq s \sigma / R^{2}$, i.e. for small $s$. Hence for small $s>0$, the operator $B_{\lambda}(s)$ is positive definite, which means that $\lambda$ cannot be an eigenvalue of (1.9), where in the third line of (1.9) $\sigma$ is replaced by $s \sigma$. On the other hand, choosing $\rho=1$ we have

$$
\left(B_{\lambda}(s) 1 \mid 1\right)_{\Sigma}=\lambda \delta|\Sigma|+\lambda l\left(T_{\lambda} 1 \mid 1\right)_{\Sigma}-s \sigma|\Sigma| / R^{2}<0
$$

if $s$ becomes large. Now we set

$$
s_{*}:=s_{*}(\lambda):=\sup \left\{s>0: B_{\lambda}(s) \text { is positive definite }\right\} .
$$

Then $B_{\lambda}\left(s_{*}\right)$ is still semi-definite, but not definite, and hence by compactness of the resolvent has a nontrivial kernel. Therefore, for a given $\lambda>0$ there is a $s_{*}=s_{*}(\lambda)$ such that $\lambda$ is an eigenvalue of (1.9) where $\sigma A_{\Sigma}$ is replaced by $s_{*} \sigma A_{\Sigma}$ in the third line.

Next we show that positive eigenvalues are simple. Rewrite (5.5) as

$$
\lambda \delta \rho_{0}+\lambda l T_{\lambda} \rho_{0}+\sigma A_{\Sigma} \rho_{0}=-\left\{\lambda \delta+\lambda l T_{\lambda} 1-\sigma / R^{2}\right\} \bar{\rho} .
$$

Since $B_{\lambda}$ is positive definite on $L_{2,0}(\Sigma)$, this equation has precisely one solution for given $\bar{\rho}$, which shows that the eigenspace $N\left(\lambda+L_{\delta}\right)$ is at most one-dimensional for any given $\lambda>0$.

To show that nontrivial eigenvalues are semi-simple, suppose that

$$
\left(\lambda+L_{\delta}\right)(v, \rho)=\left(v_{1}, \rho_{1}\right), \quad\left(\lambda+L_{\delta}\right)\left(v_{1}, \rho_{1}\right)=0 .
$$

Then by Green's formula

$$
\begin{aligned}
\left\|\sqrt{\kappa} v_{1}\right\|_{\Omega}^{2}= & \left(\lambda \kappa v-d \Delta v \mid v_{1}\right)_{\Omega} \\
= & \left(v \mid \lambda \kappa v_{1}-d \Delta v_{1}\right)_{\Omega}+\left(\left[d \partial_{\nu} v\right] \mid v_{1}\right)_{\Sigma}-\left(v \mid\left[d \partial_{\nu} v_{1}\right]\right)_{\Sigma} \\
= & (\delta / l)\left(\left[d \partial_{\nu} v\right] \mid\left[d \partial_{\nu} v_{1}\right]\right)_{\Sigma}+l \sigma\left(\lambda \rho-\rho_{1} \mid A_{\Sigma} \rho_{1}\right)_{\Sigma} \\
& -(\delta / l)\left(\left[d \partial_{\nu} v\right] \mid\left[d \partial_{\nu} v_{1}\right]\right)_{\Sigma}-\lambda l \sigma\left(A_{\Sigma} \rho \mid \rho_{1}\right)_{\Sigma}-\lambda \delta l\left\|\rho_{1}\right\|_{\Sigma}^{2} \\
= & -l \sigma\left(\rho_{1} \mid A_{\Sigma} \rho_{1}\right)-\lambda \delta l\left\|\rho_{1}\right\|_{\Sigma}^{2}
\end{aligned}
$$

which yields

$$
\left\|\sqrt{\kappa} v_{1}\right\|_{\Omega}^{2}+l \sigma\left(\rho_{1} \mid A_{\Sigma} \rho_{1}\right)+\lambda \delta l\left\|\rho_{1}\right\|_{\Sigma}^{2}=0 .
$$

It follows now from equation (4.2) that $v$ is constant on $\Omega \backslash \Sigma$. Since $\lambda \neq 0$ we then obtain from (1.9) that $\left(v_{1}, \rho_{1}\right)=(0,0)$. Thus any nontrivial eigenvalue is semi-simple, and in particular positive eigenvalues are algebraically simple.

We want to show that for $\zeta>1$ there is precisely one positive eigenvalue $\lambda_{*}>0$. For this purpose we fix the parameters $d, l, \sigma, \delta$ as well as $R$, but replace $\kappa$ by $s \kappa$ in the first line of (1.9). Fixing $\mu=\lambda s$ and scaling $\rho \mapsto \rho / s$ we obtain the scaling $\sigma \mapsto s \sigma$. The argument given above then shows that there is $s_{*}>0$ such that $\mu$ is a simple eigenvalue of the scaled problem, hence $\lambda_{*}=\mu / s_{*}>0$ is a simple positive 
eigenvalue for (1.9) with $\kappa$ replaced by $s_{*} \kappa$ in the first line. Since $\lambda_{*}=\lambda_{*}\left(s_{*}\right)$ is simple, the eigenvalue problem



has a smooth (analytic) family $\left[s \mapsto\left(\lambda_{*}(s), v(s), \rho(s)\right)\right]$ of solutions, which exists as long as $\lambda_{*}(s)$ remains a simple eigenvalue. As $\zeta(s):=s \sigma(\kappa \mid 1)_{\Omega} / l|\Sigma| R^{2}$ approaches the value $\zeta=1$ from above, we must have $\lambda_{*}(s) \rightarrow 0$ from the right. This means that at the value

$$
s=s_{0}:=\frac{l|\Sigma| R^{2}}{\sigma(\kappa \mid 1)_{\Omega}}
$$

the eigenvalue $\lambda_{*}(s)$ passes through the origin, in accordance with the jump of the algebraic multiplicity by 1 of the eigenvalue 0 for $L_{\delta}$ at $\zeta=1=\zeta\left(s_{0}\right)$. This shows that there can only be one positive eigenvalue for (5.6), independently of the values of the parameters, and there is precisely one if and only if $\zeta>1$.

If $\zeta=\sigma(\kappa \mid 1) \Omega / l|\Sigma| R^{2}>1$ then we have that $s_{0}<1$. The argument given above shows that the modified eigenvalue problem (5.6) has for each $s>s_{0}$ exactly one simple eigenvalue. This is in particular true for $s=1$, thus establishing Theorem 2.1(f).

Now we turn our attention to the case $l<0$. As before, we conclude that the operator $L_{\delta}$ has countably many eigenvalues. We note that the argument given in Section 4 also applies to the case $l<0$ and $\delta=0$, showing that all eigenvalues of (1.9) are real in this case.

In the following, we assume that $l<0$ and $\delta>0$. In order to show Theorem 1.1(f) we consider the operators $B_{\lambda}:=\lambda \delta I+\lambda l T_{\lambda}+\sigma A_{\Sigma}$ for $\lambda>0$. By Proposition 5.1 we have

$$
\left(B_{\lambda} \rho \mid \rho\right)_{\Sigma} \geq\left(\delta \lambda-|l| M_{0} \lambda^{1 / 2}-\sigma / R^{2}\right)\|\rho\|_{\Sigma}^{2} \geq\|\rho\|_{\Sigma}^{2}
$$

provided $\lambda \geq \mu_{0}$, for some $\mu_{0} \geq \lambda_{0}$. Hence $B_{\lambda}$ is positive definite for large $\lambda>0$. On the other hand we have

$$
\left(B_{\lambda} 1 \mid 1\right)_{\Sigma}=\lambda \delta|\Sigma|-\lambda|l|\left(T_{\lambda} 1 \mid 1\right)_{\Sigma}-\sigma|\Sigma| / R^{2} \leq \lambda \delta|\Sigma|-\sigma|\Sigma| / R^{2} .
$$

Thus for $\lambda$ small we see that $B_{\lambda}$ is not positive. Let

$$
\lambda_{*}:=\inf \left\{\lambda>0: B_{\mu} \text { is positive definite for all } \mu \geq \lambda\right\} .
$$

Then $B_{\lambda_{*}}$ is still semi-definite, but not definite, and hence by compactness of the resolvent has a nontrivial kernel. This shows that $\lambda_{*}$ is an eigenvalue of (1.9), proving Theorem 1.1(f).

Remarks 5.2. Suppose $l<0$ and $\delta>0$.

(a) While it is still true that all non-trivial eigenvalues of (1.9) are semi-simple, we cannot conclude that positive eigenvalues are simple. 
(b) We do not know whether all eigenvalues of (1.9) are real if $l<0$ and $\delta>0$. We can, however, prove that every sector $[|\arg \lambda| \leq \theta]$ can only contain finitely many eigenvalues for a fixed $\theta \in(0, \pi)$. This can be shown as follows. Let $\theta \in(\pi / 2, \pi)$ be fixed and suppose that $|\arg \lambda| \leq \theta$. Moreover, let $\alpha \in(0, \pi / 2)$ be an arbitrary fixed number. Then one verifies that

$$
|\lambda+\mu| \geq \min \{\sin \alpha, \sin (\pi-\theta)\}|\lambda|, \quad \text { whenever } \mu \in \mathbb{R}, \alpha \leq|\arg \lambda| \leq \theta
$$

and this shows that there exists a constant $c>0$ such that

$$
\left|\lambda \delta+\sigma\left(A_{\Sigma} \rho \mid \rho\right)_{\Sigma}\right| \geq c|\lambda|, \quad \alpha \leq|\arg \lambda| \leq \theta .
$$

Using that $\sigma\left(A_{\Sigma} \rho \mid \rho\right) \geq-\sigma / R^{2}\|\rho\|_{\Sigma}^{2}$ we see that

$$
\left|\lambda \delta+\sigma\left(A_{\Sigma} \rho \mid \rho\right)_{\Sigma}\right| \geq(\delta / 2)|\lambda|, \quad\|\rho\|_{\Sigma}=1, \quad \operatorname{Re} \lambda \geq 2 \sigma / \delta R^{2} .
$$

Combining (5.8)-(5.9) yields

$$
\left|\lambda \delta(\rho \mid \rho)_{\Sigma}+\sigma\left(A_{\Sigma} \rho \mid \rho\right)_{\Sigma}\right| \geq k|\lambda|, \quad\|\rho\|_{\Sigma}=1, \quad|\arg \lambda| \leq \theta, \quad|\lambda| \geq \eta,
$$

where $k=\min \{c, \delta / 2\}$ and $\eta=\left(2 \sigma / \delta R^{2}\right)(1 / \cos \alpha)$. Let $\lambda_{0}$ and $M_{0}$ be as in Proposition 5.1. Suppose that $\lambda \in \mathbb{C} \backslash\{0\}$ with $[|\arg \lambda| \leq \theta]$ is an eigenvalue of (1.9) with eigenfunction $(v, \rho)$. Then we have

$$
\lambda \delta \rho+\sigma A_{\Sigma} \rho=\lambda|l| T_{\lambda} \rho
$$

and we can assume without loss of regularity that $\|\rho\|_{\Sigma}=1$. If $|\lambda| \geq \max \left\{\lambda_{0}, \eta\right\}$ then we conclude from (5.10)-(5.11) and Proposition 5.1 that

$$
k|\lambda| \leq M_{0}|l||\lambda|^{1 / 2}
$$

and so $|\lambda|$ is bounded by $\left(M_{0} l / k\right)^{2}$. Clearly, if $|\lambda| \leq \max \left\{\lambda_{0}, \eta\right\}$ we have a trivial bound. This shows that all possible eigenvalues in the sector $[|\arg \lambda| \leq \theta]$ are bounded. Since eigenvalues cannot accumulate in a bounded set, we see that (1.9) can only have finitely many eigenvalues in the sector $[|\arg \lambda| \leq \theta]$.

\section{Analysis of the Unstable Eigenvalue}

In this section we analyze the properties of the unstable eigenvalue $\lambda_{*}$ of problem (5.6) in case $l>0$ in more detail. In particular, we study the behaviour of $\lambda_{*}(s)$ and the corresponding eigenfunctions near the critical value 1 of $\zeta(s)$, i.e. for $s$ near $s_{0}$, see equation (5.7).

\section{Proof Theorem 1.3.}

(a) We will first analyze the behavior of $\left(\lambda_{*}(s), v(s), \rho(s)\right)$ for $s$ near $s_{0}$. In order to do so we use the following ansatz:

$$
\begin{aligned}
& \lambda_{*}(s)=\left(s-s_{0}\right) \lambda_{1}(s), \\
& v(s)=-1+\left(s-s_{0}\right) \lambda_{1}(s) v_{1}(s), \\
& \rho(s)=\rho_{0}+\left(s-s_{0}\right) \eta+\left(s-s_{0}\right) \lambda_{1}(s)\left(\rho_{1}(s)+\vec{\beta}(s) \cdot \vec{y}\right), \\
& \left(v_{1}(s) \mid \kappa\right)_{\Omega}=0, \quad\left(\rho_{1}(s) \mid 1\right)_{\Sigma}=\left(\rho_{1}(s) \mid Y_{j}\right)=0,1 \leq j \leq n,
\end{aligned}
$$


with

$$
\rho_{0}:=R^{2} / \sigma+\vec{\alpha} \cdot \vec{y}, \quad \eta:=(\kappa \mid 1)_{\Omega} / l \Sigma,
$$

where $\vec{\alpha}, \vec{\beta}(s) \in \mathbb{R}^{n}$ and $\vec{y}=\left(Y_{1}, \ldots, Y_{n}\right)$. Setting $r=s-s_{0}$ and inserting this ansatz into the eigenvalue problem (5.6) we obtain the following system of equations:

$$
\left\{\begin{aligned}
-d \Delta v_{1} & =s_{0} \kappa+r \kappa\left(1-s \lambda_{1} v_{1}\right) & & \text { in } \quad \Omega \backslash \Sigma \\
\partial_{\nu} v_{1} & =0 & & \text { on } \quad \partial \Omega \\
{\left[v_{1}\right] } & =0 & & \text { on } \Sigma \\
-\left[d \partial_{\nu} v_{1}\right] & =-l \rho_{0}-r l \eta-r \lambda_{1} l\left(\rho_{1}+\vec{\beta} \cdot \vec{y}\right) & & \text { on } \Sigma \\
\sigma A_{\Sigma} \rho_{1} & =\frac{\sigma \eta}{R^{2} \lambda_{1}}-\delta \rho_{0}+v_{1}-r \delta \eta-r \lambda_{1} \delta\left(\rho_{1}+\vec{\beta} \cdot \vec{y}\right) & & \text { on } \Sigma .
\end{aligned}\right.
$$

We first observe that due to (5.7), (6.1) ${ }_{4}$ and (6.2), and the fact that $\left(Y_{i} \mid 1\right)_{\Sigma}=0$, the compatibility condition

$$
\left(1 \mid s_{0} \kappa+r \kappa\left(1-s \lambda_{1} v_{1}\right)\right)_{\Omega}-l\left(1 \mid \rho_{0}+r \eta+r \lambda_{1}\left(\rho_{1}+\vec{\beta} \cdot \vec{y}\right)\right)_{\Sigma}=0
$$

holds. It is our intention to apply the implicit function therorem to find a smooth (analytic) curve of solutions

$$
\left[s \mapsto\left(\lambda_{1}(s), v_{1}(s), \rho_{1}(s), \vec{\beta}(s)\right)\right]
$$

of (6.3) for $s$ near $s_{0}$. The idea is to use the $(n+1)$ orthogonality conditions

$$
\left(A_{\Sigma} \rho_{1} \mid 1\right)=\left(A_{\Sigma} \rho_{1} \mid Y_{j}\right)=0, \quad 1 \leq j \leq n,
$$

to determine the $(n+1)$ scalar functions $\lambda_{1}$ and $\beta_{j}$. In order to do so, we will first derive an expression for $\vec{\alpha}$ and $\vec{\beta}$. Taking the inner product of $(6.3)_{5}$ with $Y_{j}$ yields

$$
0=-\delta \vec{\alpha}+\left(v_{1} \mid \vec{y}\right)_{\Sigma}-r \delta \lambda_{1} \vec{\beta}
$$

where $\left(v_{1} \mid \vec{y}\right)_{\Sigma}$ denotes the vector in $\mathbb{R}^{n}$ with components $\left(v_{1} \mid Y_{j}\right)_{\Sigma}, 1 \leq j \leq n$. Due to Proposition 3.2 we have

$$
\begin{aligned}
\left(v_{1} \mid Y_{j}\right)_{\Sigma} & =\left(v_{1} \mid-\left[d \partial_{\nu} T_{0} Y_{j}\right]\right)_{\Sigma}=\int_{\Omega} d \operatorname{div}\left(v_{1} T_{0} Y_{j}\right) d x \\
& =\left(d \nabla v_{1} \mid \nabla T_{0} Y_{j}\right)_{\Omega}=\left(-d \Delta v_{1} \mid T_{0} Y_{j}\right)_{\Omega}+\left(-\left[d \partial_{\nu} v_{1}\right] \mid T_{0} Y_{j}\right)_{\Sigma} \\
& =\left(s_{0} \kappa+r \kappa\left(1-s \lambda_{1} v_{1}\right) \mid T_{0} Y_{j}\right)_{\Omega}-l\left(\rho_{0}+r \eta+r \lambda_{1}\left(\rho_{1}+\vec{\beta} \cdot \vec{y}\right) \mid T_{0} Y_{j}\right)_{\Sigma} . \\
& =-r s \lambda_{1}\left(\kappa v_{1} \mid T_{0} Y_{j}\right)_{\Omega}-l\left(\rho_{0}+r \eta+r \lambda_{1}\left(\rho_{1}+\vec{\beta} \cdot \vec{y}\right) \mid T_{0} Y_{j}\right)_{\Sigma}
\end{aligned}
$$

for $j=1, \cdots, n$. Setting first $r=0$ yields an equation for $\vec{\alpha}$, namely

$$
0=-\delta \vec{\alpha}-\left(l R^{2} / \sigma\right)\left(1 \mid T_{0} \vec{y}\right)_{\Sigma}-l\left(T_{0} \vec{y} \mid \vec{y}\right)_{\Sigma} \vec{\alpha},
$$

i.e.

$$
\vec{\alpha}=-\left(\delta I+l\left(T_{0} \vec{y} \mid \vec{y}\right)_{\Sigma}\right)^{-1}\left(l R^{2} / \sigma\right)\left(1 \mid T_{0} \vec{y}\right)_{\Sigma},
$$


where $\left(T_{0} \vec{y} \mid \vec{y}\right)_{\Sigma}$ denotes the symmetric matrix with entries $\left[\left(T_{0} Y_{i} \mid Y_{j}\right)_{\Sigma}\right]_{1 \leq i, j \leq n}$. Here we remind that

$$
\left\langle\left(T_{0} \vec{y} \mid \vec{y}\right)_{\Sigma} \xi \mid \xi\right\rangle=\sum_{i, j=1}^{n} \xi_{i} \xi_{j}\left(T_{0} Y_{i} \mid Y_{j}\right)_{\Sigma}=\left(T_{0}(\xi \cdot \vec{y}) \mid(\xi \cdot \vec{y})\right)_{\Sigma} \geq c\|\xi\|^{2}
$$

for $\xi \in \mathbb{R}^{n}$, where $\langle\cdot \mid \cdot\rangle$ denotes the Euclidean inner product on $\mathbb{R}^{n}$. This shows that the matrix $\left(T_{0} \vec{y} \mid \vec{y}\right)_{\Sigma}$ is positive definite, and hence $\delta I+l\left(T_{0} \vec{y} \mid \vec{y}\right)_{\Sigma}$ is invertible for any $\delta \geq 0$. Next we obtain for $\vec{\beta}$

$$
\vec{\beta}=-\left(\delta I+l\left(T_{0} \vec{y} \mid \vec{y}\right)_{\Sigma}\right)^{-1}\left\{s\left(\kappa v_{1} \mid T_{0} \vec{y}\right)_{\Omega}+l\left(\eta / \lambda_{1}+\rho_{1} \mid T_{0} \vec{y}\right)_{\Sigma}\right\} .
$$

Thus we have a function $\vec{\beta}=\vec{\beta}\left(\lambda_{1}, v_{1}, \rho_{1}, s\right)$. Finally, we obtain an equation for $\lambda_{1}$ by taking the inner product of $(6.3)_{5}$ with 1

$$
0=\sigma \eta|\Sigma| / R^{2} \lambda_{1}-\delta R^{2}|\Sigma| / \sigma+\left(v_{1} \mid 1\right)_{\Sigma}-r \delta|\Sigma| \eta .
$$

Employing the relation $\left(\kappa \mid v_{1}\right)_{\Omega}=0$ and equations (6.3) $)_{1,4}$ as well as (6.5) yields

$$
\begin{array}{rl}
-r & s \lambda_{1}\left(\kappa v_{1} \mid v_{1}\right)_{\Omega}=\left(s_{0} \kappa+r \kappa\left(1-s \lambda_{1} v_{1}\right) \mid v_{1}\right)_{\Omega} \\
\quad & =\left(-d \Delta v_{1} \mid v_{1}\right)_{\Omega}=\left\|\sqrt{d} \nabla v_{1}\right\|_{\Omega}^{2}+\left(\left[d \partial_{\nu} v_{1}\right] \mid v_{1}\right)_{\Sigma} \\
& =\left\|\sqrt{d} \nabla v_{1}\right\|_{\Omega}^{2}+\left(l \rho_{0}+r l \eta+r \lambda_{1} l\left(\rho_{1}+\vec{\beta} \cdot \vec{y}\right) \mid v_{1}\right)_{\Sigma} \\
& =\left\|\sqrt{d} \nabla v_{1}\right\|_{\Omega}^{2}+\left\{l R^{2} / \sigma+r l \eta\right\}\left(v_{1} \mid 1\right)_{\Sigma}+l \delta\left|\vec{\alpha}+r \lambda_{1} \vec{\beta}\right|^{2}+r \lambda_{1} l\left(\rho_{1} \mid v_{1}\right)_{\Sigma} .
\end{array}
$$

This leads to

$$
\begin{aligned}
0= & \left\|\sqrt{d} \nabla v_{1}\right\|_{\Omega}^{2}-\left\{l R^{2} / \sigma+r l \eta\right\}\left\{\left(\sigma|\Sigma| \eta / R^{2} \lambda_{1}\right)-\delta R^{2}|\Sigma| / \sigma-r \delta|\Sigma| \eta\right\} \\
& +l \delta\left|\vec{\alpha}+r \lambda_{1} \vec{\beta}\right|^{2}+r \lambda_{1}\left\{l\left(\rho_{1} \mid v_{1}\right)_{\Sigma}+s\left(\kappa v_{1} \mid v_{1}\right)_{\Omega}\right\},
\end{aligned}
$$

where we used equation (6.9).

Suppose now that $v_{1}$ solves the first four equations of (6.3). Then one easily verifies that equation (6.5) is equivalent to (6.6) \& (6.8). Moreover, assuming once again that $v_{1}$ satisfies the first four equations of (6.3), and that $\vec{\alpha}$ and $\vec{\beta}$ satisfy (6.6) and (6.8) one verifies that

$$
(6.9) \Longleftrightarrow(6.10) \text {. }
$$

For $r=0$, that is for $s=s_{0}$, we obtain from (6.10)

$$
\lambda_{1}\left(s_{0}\right)=l \Sigma \eta /\left\{\left\|\sqrt{d} \nabla v_{1}\left(s_{0}\right)\right\|_{\Omega}^{2}+l \delta\left(|\vec{\alpha}|^{2}+R^{4}|\Sigma| / \sigma^{2}\right)\right\},
$$

where $v_{1}\left(s_{0}\right)$ is the unique solution of problem (3.4) with $(f, g)=\left(s_{0} \kappa,-l \rho_{0}\right)$ and $\left(\kappa \mid v_{1}\left(s_{0}\right)\right)_{\Omega}=0$, see Proposition 3.2. This shows that $\lambda_{1}\left(s_{0}\right)$ is uniquely defined and strictly positive. Moreover, we also know from (6.11) that

$$
0=\left(\sigma \eta|\Sigma| / R^{2} \lambda_{1}\left(s_{0}\right)\right)-\delta R^{2}|\Sigma| / \sigma+\left(v_{1}\left(s_{0}\right) \mid 1\right)_{\Sigma}-r \delta|\Sigma| \eta .
$$

We obtain $\rho_{1}\left(s_{0}\right)$ by solving the equation

$$
\sigma A_{\Sigma} \rho_{1}=\sigma \eta / R^{2} \lambda_{1}\left(s_{0}\right)-\delta \rho_{0}+v_{1}\left(s_{0}\right)
$$


for $\rho_{1}$, which is possible since we chose $\lambda_{1}\left(s_{0}\right)$ and $\vec{\alpha}$ in such a way that the necessary orthogonality conditions of Proposition 3.1.(d) hold.

Equation (6.8) shows that the mapping

$$
\begin{aligned}
& I \times\left\{v \in W_{2}^{2}(\Omega \backslash \Sigma):[v]=0 \text { on } \Sigma\right\} \times W_{2}^{2}(\Sigma) \times \mathbb{R} \rightarrow \mathbb{R}^{n}, \\
& {\left[\left(\lambda_{1}, v_{1}, \rho_{1}, s\right) \mapsto \vec{\beta}\left(\lambda_{1}, v_{1}, \rho_{1}, s\right)\right] \text { is analytic }}
\end{aligned}
$$

where $I \subset \mathbb{R}$ is an open interval that contains $\lambda_{1}\left(s_{0}\right)$ but does not contain 0 .

We are now in a position to apply the implicit function theorem at the point $\left(v_{1}\left(s_{0}\right), \rho_{1}\left(s_{0}\right), s_{0}\right)$ to solve the first four equations in (6.3) and equation (6.9) for $\left(\lambda_{1}, v_{1}\right)$ in terms of $\left(\rho_{1}, s\right)$. We choose the following functional analytic setting

$$
\begin{aligned}
X_{1} & :=\left\{v \in W_{2}^{2}(\Omega \backslash \Sigma): \partial_{\nu} v=0 \text { on } \partial \Omega,[v]=0 \text { on } \Sigma,(\kappa \mid v)_{\Omega}=0\right\}, \\
X_{2} & :=\left\{\rho \in W_{2}^{2}(\Sigma):(\rho \mid 1)_{\Sigma}=\left(\rho \mid Y_{j}\right)_{\Sigma}=0,1 \leq j \leq n\right\}, \\
X & :=\mathbb{R} \times X_{1} \times X_{2} \times \mathbb{R}, \\
Y & :=\mathbb{R} \times\left\{(f, g) \in L_{2}(\Omega) \times W_{2}^{1 / 2}(\Sigma):(f \mid 1)_{\Omega}+(g \mid 1)_{\Sigma}=0\right\}
\end{aligned}
$$

and we define $F: V \subset X \rightarrow Y$ by means of

$$
F\left(\lambda_{1}, v_{1}, \rho_{1}, s\right):=\left(\begin{array}{l}
\sigma \eta|\Sigma| / R^{2} \lambda_{1}-\delta R^{2}|\Sigma| / \sigma+\left(v_{1} \mid 1\right)_{\Sigma}-r \delta|\Sigma| \eta \\
-d \Delta v_{1}-s_{0} \kappa-r \kappa\left(1-s \lambda_{1} v_{1}\right) \\
-\left[d \partial_{\nu} v_{1}\right]+l\left(\rho_{0}+r \eta+r \lambda_{1}\left(\rho_{1}+\vec{\beta} \cdot \vec{y}\right)\right)
\end{array}\right)
$$

where $V:=I \times X_{1} \times X_{2} \times \mathbb{R}$.

Equation (6.4) implies that $F$ maps $V$ into $Y$, and (6.13) and the definition of $F$ shows that

$$
\left[\left(\lambda_{1}, v_{1}, \rho_{1}, s\right) \mapsto F\left(\lambda_{1}, v_{1}, \rho_{1}, s\right)\right] \in C^{\omega}(V, Y) .
$$

Clearly, the first four equations of (6.3) together with equation (6.9) are equivalent to $F\left(\lambda_{1}, v_{1}, \rho_{1}, s\right)=(0,0,0)$. Since we already know that

$$
F\left(\lambda_{1}\left(s_{0}\right), v_{1}\left(s_{0}\right), \rho_{1}\left(s_{0}\right), s_{0}\right)=(0,0,0)
$$

we are left with verifying that the derivative of $F$ at the point $\left(v_{1}\left(s_{0}\right), \rho_{1}\left(s_{0}\right), s_{0}\right)$ w.r.t. $\left(\rho_{1}, v_{1}\right)$ is an isomorphism, i.e.

$$
\mathbb{D}_{1} F\left(\lambda_{1}\left(s_{0}\right), v_{1}\left(s_{0}\right), \rho_{1}\left(s_{0}\right), s_{0}\right) \in \operatorname{Isom}\left(\mathbb{R} \times X_{1}, Y\right) .
$$

If follows from Proposition 3.2.(a) that the problem

$$
\mathbb{D}_{1} F\left(\lambda_{1}\left(s_{0}\right), v_{1}\left(s_{0}\right), \rho_{1}\left(s_{0}\right), s_{0}\right)(\lambda, w)=(\mu, f, g)
$$

has for each $(\mu, f, g) \in Y$ a unique solution $(\lambda, w) \in \mathbb{R} \times X_{1}$, namely

$$
w=R_{0}(f, g), \quad \lambda=\frac{R^{2} \lambda_{1}^{2}\left(s_{0}\right)}{\sigma \eta|\Sigma|}\left(\left(R_{0}(f, g) \mid 1\right)_{\Sigma}-\mu\right),
$$

where $w=R_{0}(f, g)$ is the unique solution of (3.4) with $(\kappa \mid w)_{\Omega}=0$. 
The implicit function theorem then yields a neighborhood $U$ of $\left(\rho_{1}\left(s_{0}\right), s_{0}\right)$ in $X_{2} \times \mathbb{R}$ such that

$$
\begin{aligned}
& {\left[\left(\rho_{1}, s\right) \mapsto\left(\lambda_{1}\left(\rho_{1}, s\right), v_{1}\left(\rho_{1}, s\right)\right] \in C^{\omega}\left(U, \mathbb{R} \times X_{1}\right)\right.} \\
& F\left(\lambda_{1}\left(\rho_{1}, s\right) v_{1}\left(\rho_{1}, s\right), \rho_{1}, s\right)=(0,0,0), \quad\left(\rho_{1}, s\right) \in U .
\end{aligned}
$$

Combining all the above results we conclude that

$$
\left[\left(\rho_{1}, s\right) \mapsto\left(\lambda_{1}\left(\rho_{1}, s\right), v_{1}\left(\rho_{1}, s\right), \vec{\beta}\left(\rho_{1}, s\right)\right)\right] \in C^{\omega}\left(U, \mathbb{R} \times X_{1} \times \mathbb{R}^{n}\right)
$$

and that the functions $\left(\lambda_{1}\left(\rho_{1}, s\right), v_{1}\left(\rho_{1}, s\right), \vec{\alpha}, \vec{\beta}\left(\rho_{1}, s\right)\right)$ satisfy the first four equations of (6.3) as well as (6.5) and (6.9). We now insert these functions into the equation for $\rho_{1}$ which gives an equation of the form

$$
G\left(\rho_{1}, s\right):=\sigma A_{\Sigma} \rho_{1}-\sigma \eta / R^{2} \lambda_{1}\left(s_{0}\right)+\delta \rho_{0}-v_{1}\left(s_{0}\right)-\left(s-s_{0}\right) R\left(\rho_{1}, s\right)=0,
$$

where $\left[\left(\rho_{1}, s\right) \mapsto R\left(\rho_{1}, s\right)\right] \in C^{\omega}\left(U, L_{2}(\Sigma)\right)$. By (6.5) and (6.9) we know that

$$
G: X_{2} \times \mathbb{R} \rightarrow Z:=\left\{g \in L_{2}(\Sigma):(g \mid 1)_{\Sigma}=\left(g \mid Y_{j}\right)=0,1 \leq j \leq n\right\} .
$$

Moreover, we also know that $G\left(\rho_{1}\left(s_{0}\right), s_{0}\right)=0$. The derivative of $G$ with respect to $\rho_{1}$ at $\left(\rho_{1}\left(s_{0}\right), s_{0}\right)$ is $\sigma A_{\Sigma}$, and Proposition 3.1.(d) and the implicit function theorem then yield a analytic curve

$$
\left[s \mapsto \rho_{1}(s)\right] \in C^{\omega}\left(\left(s_{0}-\varepsilon_{0}, s_{0}+\varepsilon_{0}\right), X_{2}\right)
$$

such that $G\left(\rho_{1}(s), s\right)=0$.

Combining all the results we obtain an analytic curve of solutions

$$
\left[s \mapsto\left(\lambda_{*}(s), v(s), \rho(s)\right)\right]
$$

of (5.6) for $s \in\left(s_{0}-\varepsilon_{0}, s_{0}+\varepsilon_{0}\right)$. If $s>s_{0}$ then the statement in (a) follows from the considerations in Section 5 .

(b) The proof of part (a) shows that the eigenvalue curve $\left[s \mapsto \lambda_{*}(s)\right]$ is analytic near the critical value $s=s_{0}$ and crosses the imaginary axis at $s=s_{0}$ with positive speed $\lambda_{1}\left(s_{0}\right)$, see $(6.12)$.

(c) If the stability condition (5.1) is violated, then we can conclude from the identity (4.4), where $\kappa$ is now replaced with $s \kappa$ and $\lambda$ is replaced with $\lambda_{*}$, that

$$
\left(\lambda_{*}(s) l \delta|\Sigma|+\frac{l^{2}|\Sigma|^{2}}{s(\kappa \mid 1)_{\Omega}}-\frac{l \sigma|\Sigma|}{R^{2}}\right) \leq 0,
$$

and this shows that $\lambda_{*}(s) \leq \frac{\sigma}{\delta R^{2}}(1-1 / \zeta(s))$ provided $\delta>0$, i.e. if kinetic undercooling is present.

In case $\delta>0$, i.e. in the presence of kinetic undercooling, the eigenvalue $\lambda_{*}(s)$ stays bounded as $s \rightarrow \infty$. In contrast to this, we will show that $\lambda_{*}(s) \rightarrow \infty$ as $s \rightarrow \infty$ in case $\delta=0$.

(d) First we show that $\lambda_{*}(s)$ is strictly increasing. To see this, we differentiate (5.6) 
w.r.t. $s$ and form the inner product of the resulting equation in $\Omega$ with $v=v(s)$. This yields with Green's formula

$$
\begin{aligned}
-\left(s \lambda_{*}(s)\right)^{\prime}\|\sqrt{\kappa} v\|_{\Omega}^{2}= & s \lambda_{*}\left(\kappa v^{\prime} \mid v\right)_{\Omega}-\left(d \Delta v^{\prime} \mid v\right)_{\Omega} \\
= & \left(v^{\prime} \mid s \lambda_{*} \kappa v-d \Delta v\right)_{\Omega}+\left(\left[d \partial_{\nu} v^{\prime}\right] \mid v\right)_{\Sigma}-\left(v^{\prime} \mid\left[d \partial_{\nu} v\right]\right)_{\Sigma} \\
= & (\delta / l)\left(\left[d \partial_{\nu} v^{\prime}\right] \mid\left[d \partial_{\nu} v\right]\right)_{\Sigma}+\left(\lambda_{*} l \rho^{\prime}+\lambda_{*}^{\prime} l \rho \mid \sigma A_{\Sigma} \rho\right)_{\Sigma} \\
& -(\delta / l)\left(\left[d \partial_{\nu} v\right] \mid\left[d \partial_{\nu} v^{\prime}\right]\right)_{\Sigma}-\left(\lambda_{*} l \rho \mid \sigma A_{\Sigma} \rho^{\prime}\right)_{\Sigma} \\
= & \lambda_{*}^{\prime} l \sigma\left(A_{\Sigma} \rho \mid \rho\right)_{\Sigma},
\end{aligned}
$$

hence

$$
\lambda_{*}\|\sqrt{\kappa} v\|_{\Omega}^{2}+\lambda_{*}^{\prime}\left\{\|\sqrt{s \kappa} v\|_{\Omega}^{2}+l \sigma\left(A_{\Sigma} \rho \mid \rho\right)_{\Sigma}\right\}=0 .
$$

Employing (4.1) once more (with $\kappa$ replaced by $s \kappa$ ) we obtain

$$
\lambda_{*}^{\prime}(s)=\lambda_{*}^{2}(s)\|\sqrt{\kappa} v\|_{\Omega}^{2} /\left\{\|\sqrt{d} \nabla v\|_{\Omega}^{2}+(\delta / l)\left\|\left[d \partial_{\nu} v\right]\right\|_{\Sigma}^{2}\right\},
$$

which yields $\lambda_{*}^{\prime}(s)>0$ for $s \neq s_{0}$. If $s=s_{0}$ then we have already established in (b) that $\lambda_{*}^{\prime}\left(s_{0}\right)>0$, and this shows the assertion of Theorem 1.3.(c).

(e) To show that $\lambda_{*}(s) \rightarrow \infty$ as $s \rightarrow \infty$ in case $\delta=0$, we employ the estimate in Proposition 5.1.(b). We first observe that due to the fact that $\lambda_{*}(s)$ is increasing in $s$, there exists a number $s_{1}>s_{0}$ such that $s \lambda_{*}(s) \geq \lambda_{0}$ for $s \geq s_{1}$, where $\lambda_{0}$ is the number occurring in Proposition 5.1.(b). It then follows from the relation $\lambda_{*} T_{s \lambda_{*}} \rho+\sigma A_{\Sigma} \rho=0$ that

$$
\begin{aligned}
\sigma^{2}\left\|A_{\Sigma} \rho\right\|_{\Sigma}^{2}=\sigma^{2}\left\|A_{\Sigma} \rho_{0}\right\|_{\Sigma}^{2}+\left(\sigma^{2}|\Sigma| / R^{2}\right) \bar{\rho}^{2} & =\lambda_{*}^{2}\left\|T_{s \lambda_{*}} \rho\right\|_{\Sigma}^{2} \\
& \leq M_{1}\|\rho\|_{\Sigma}^{2} \lambda_{*}(s) / s, \quad s \geq s_{1} .
\end{aligned}
$$

Multiplying the eigenvalue problem (5.6) with $\left(s \lambda_{*} v-(d / \kappa) \Delta v\right)$ and using the divergence theorem and (6.16) we get

$$
\begin{gathered}
\left(s \lambda_{*}\right)^{2}\|\sqrt{\kappa} v\|_{\Omega}^{2}+2 s \lambda_{*}\|\sqrt{d} \nabla v\|_{\Omega}^{2}+\|(d / \sqrt{\kappa}) \Delta v\|_{\Omega}^{2}+2 s \lambda_{*}^{2} l \sigma\left(\rho_{0} \mid A_{\Sigma} \rho_{0}\right)_{\Sigma} \\
=\left(2 s \lambda_{*}^{2} l \sigma|\Sigma| / R^{2}\right) \bar{\rho}^{2} \leq M_{2}\|\rho\|_{\Sigma}^{2} \lambda_{*}^{3}, \quad s \geq s_{1} .
\end{gathered}
$$

The relation $\lambda_{*} l \rho=\left[d \partial_{\nu} v\right]$ and the inequality above yields

$$
\begin{aligned}
\lambda_{*}^{2}\|\rho\|_{\Sigma}^{2} & =(1 / l)^{2}\left\|\left[d \partial_{\nu} v\right]\right\|_{\Sigma}^{2} \leq C\|v\|_{W_{2}^{3 / 2+\varepsilon}(\Omega \backslash \Sigma)}^{2} \leq C\|v\|_{\Omega}^{(1 / 2-\varepsilon)}\|v\|_{W_{2}^{2}(\Omega \backslash \Sigma)}^{3 / 2+\varepsilon} \\
& \leq C\left\{\|v\|_{\Omega}^{2}+\|v\|_{\Omega}^{(1 / 2-\varepsilon)}\|\Delta v\|_{\Omega}^{3 / 2+\varepsilon}\right\} \leq C\|\rho\|_{\Sigma}^{2} \lambda_{*}^{3}\left\{\frac{1}{\left(s \lambda_{*}\right)^{2}}+\frac{1}{\left(s \lambda_{*}\right)^{1 / 2-\varepsilon}}\right\} \\
& \leq C\|\rho\|_{\Sigma}^{2} \lambda_{*}^{3} /\left(s \lambda_{*}\right)^{1 / 2-\varepsilon}=C\|\rho\|_{\Sigma}^{2} \lambda_{*}^{5 / 2+\varepsilon} / s^{1 / 2-\varepsilon}
\end{aligned}
$$

for $s \geq s_{1}$, where $C$ is a generic constant that may change from line to line. Dividing by $\lambda_{*}^{2}$ and by $\|\rho\|_{\Sigma}^{2}$ this implies

$$
\lambda_{*}(s) \geq c s^{(1-2 \varepsilon) /(1+2 \varepsilon)}, \quad s \geq s_{1} .
$$

Thus we can conclude that $\liminf _{s \rightarrow \infty} \lambda_{*}(s) / s^{\theta}=\infty$ for each $\theta<1$. 
Acknowledgment: The authors would like to thank Rico Zacher for helpful discussions.

\section{REFERENCES}

[1] B.V. Bazalii, Stefan problem for the Laplace equation with regard to the curvature of the free boundary. Ukrainian Math. J. 49 (1997), 1465-1484.

[2] G. Caginalp, An analysis of a phase field model of a free boundary. Arch. Rational Mech. Anal. 92 (1986), 205-245.

[3] B. Chalmers, Principles of solidification. Krieger, Huntington, N.Y., 1977.

[4] X. Chen, The Hele-Shaw problem and area-preserving curve-shortening motion, Arch. Rational Mech. Anal. 123 (1993), 117-151.

[5] X. Chen, F. Reitich, Local existence and uniqueness of solutions of the Stefan problem with surface tension and kinetic undercooling. J. Math. Anal. Appl. 164 (1992), 350-362.

[6] X. Chen, J. Hong, F. Yi, Existence, uniqueness, and regularity of classical solutions of the Mullins-Sekerka problem. Comm. Partial Differential Equations 21 (1996), 1705-1727.

[7] R. Denk, J. Prüss, R. Zacher, Maximal $L_{p}$-regularity of parabolic problems with boundary conditions of relaxation type. In preparation.

[8] J. Escher, G. Simonett, On Hele-Shaw models with surface tension. Math. Res. Lett. 3, 467-474 (1996).

[9] J. Escher, G. Simonett, Classical solutions for the quasi-stationary Stefan problem with surface tension. Differential equations, asymptotic analysis, and mathematical physics, (Potsdam, 1996), Math. Res., 100, Akademie Verlag, Berlin, 1997, 98-104.

[10] J. Escher, G. Simonett, Classical solutions for Hele-Shaw models with surface tension. Adv. Differential Equations 2 (1997), 619-642.

[11] J. Escher, G. Simonett, A center manifold analysis for the Mullins-Sekerka model. J. Differential Equations 143 (1998), 267-292.

[12] J. Escher, J. Prüss, G. Simonett, On the Stefan problem with surface tension. Elliptic and parabolic problems (Rolduc/Gaeta, 2001), World Sci. Publ., River Edge, NJ, 2002, 377-388.

[13] J. Escher, J. Prüss, G. Simonett, Analytic solutions for a Stefan problem with GibbsThomson correction. J. Reine Angew. Math. 563 (2003), 1-52.

[14] A. Friedman, F. Reitich, The Stefan problem with small surface tension. Trans. Amer. Math. Soc. 328 (1991), 465-515.

[15] A. Friedman, F. Reitich, Nonlinear stability of a quasi-static Stefan problem with surface tension: a continuation approach. Ann. Scuola Norm. Sup. Pisa Cl. Sci. (4) 30 (2001), 341-403.

[16] M.E. Gurtin, On the two phase problem with interfacial energy and entropy. Arch. Rational Mech. Anal. 96 (1986), 199-241.

[17] M.E. Gurtin, Multiphase thermomechanics with interfacial structure. Arch. Rational Mech. Anal. 104 (1988), 195-221.

[18] E.I. Hanzawa, Classical solutions of the Stefan problem. Tôhoku Math. Jour. 33 (1981), 297-335.

[19] P. Hartman, Crystal growth: An introduction. North-Holland, Amsterdam, 1973.

[20] C. Kneisel, Über das Stefan-Problem mit Oberflächenspannung und thermischer Unterkühlung, in preparation.

[21] J.S. Langer, Instabilities and pattern formation in crystal growth. Rev. Mod. Phys. 52 (1980), 1-28.

[22] S. Luckhaus, Solutions for the two-dimensional Stefan problem with the Gibbs-Thomson law for melting temperature, European J. Appl. Math. 1 (1990), 101-111. 
[23] A.M. Meirmanov, The Stefan problem with surface tension in the three dimensional case with spherical symmetry: non-existence of the classical solution. European J. Appl. Math. 5 (1994), 1-20.

[24] W.W. Mullins, Thermodynamic equilibrium of a crystal sphere in a fluid. J. Chem. Phys. 81 (1984), 1436-1442.

[25] W.W. Mullins, R.F. Sekerka, Stability of a planar interface during solidification of a dilute binary alloy. J. Appl. Phys. 35 (1964), 444-451.

[26] J. Prüss, G. Simonett, Smooth solutions for two-phase Stefan problems with surface tension. In preparation.

[27] J. Prüss, G. Simonett, R. Zacher, Convergence of solutions to equilibria for nonlinear parabolic problems. In preparation.

[28] E. Radkevitch, The Gibbs-Thompson correction and conditions for the existence of a classical solution of the modified Stefan problem. Dokl. Akad. Nauk SSSR 316 (1991), 1311-1315; translation in Soviet Math. Dokl. 43 (1991), 274-278.

[29] E. Radkevitch, Conditions for the existence of a classical solution of a modified Stefan problem (the Gibbs-Thomson law). Mat. Sb. 183 (1992), 77-101; translation in Russian Acad. Sci. Sb. Math. 75 (1993), 221-246.

[30] H. Triebel, Höhere Analysis. (German) Hochschulbücher für Mathematik, Band 76. VEB Deutscher Verlag der Wissenschaften, Berlin, 1972.

[31] A. Visintin, Models for supercooling and superheating effects. Pitman Research Notes in Math. 120, Longman Sci.\& Tech., Essex, 1995, 200-207.

[32] A. Visintin, Models of Phase Transitions. Progress in Nonlinear Differential Equations and Their Applications, Volume 28, Birkhäuser, Boston, 1996.

[33] W. Yu, A quasisteady Stefan problem with curvature correction and kinetic undercooling. J. Partial Differential Equations 9 (1996), 55-70.

Institut für Mathematik, Martin-Luther-Universität Halle-Wittenberg，D-60120 Halle, Germany

E-mail address: jan.pruess@mathematik. uni-halle.de

Department of Mathematics, Vanderbilt University, Nashville, TN 37240, USA

E-mail address: simonett@math.vanderbilt.edu 